\title{
Combination anti-PD-1 and antiretroviral therapy provides therapeutic benefit against SIV
}

Geetha H. Mylvaganam, ${ }^{1,2}$ Lynette S. Chea, ${ }^{1,2}$ Gregory K. Tharp, ${ }^{2}$ Sakeenah Hicks, ${ }^{1,2}$ Vijayakumar Velu, ${ }^{1,2}$ Smita S. Iyer, ${ }^{1,2}$ Claire Deleage, ${ }^{3}$ Jacob D. Estes, ${ }^{3}$ Steven E. Bosinger, ${ }^{2}$ Gordon J. Freeman, ${ }^{4}$ Rafi Ahmed, ${ }^{1,2}$ and Rama R. Amara ${ }^{1,2}$

'Department of Microbiology and Immunology, Emory University School of Medicine, Atlanta, Georgia, USA. 'Division of Microbiology and Immunology, Emory Vaccine Center, Yerkes National Primate Research Center, Emory University, Atlanta, Georgia, USA. ${ }^{3}$ AIDS and Cancer Virus Program, Frederick National Laboratory for Cancer Research, Leidos Biomedical Research, Inc., Frederick, Maryland, USA. ${ }^{4}$ Department of Medical Oncology and Cancer Vaccine Center, Dana-Farber Cancer Institute, Boston, Massachusetts, USA.

Therapeutic strategies that augment antiviral immunity and reduce the viral reservoir are critical to achieving durable remission of HIV. The coinhibitory receptor programmed death-1 (PD-1) regulates CD8 ${ }^{+} \mathrm{T}$ cell dysfunction during chronic HIV and SIV infections. We previously demonstrated that in vivo blockade of PD-1 during chronic SIV infection improves the function of antiviral CD8 ${ }^{+} \mathrm{T}$ cells and B cells. Here, we tested the immunological and virological effects of PD-1 blockade combined with antiretroviral therapy (ART) in rhesus macaques. Administration of anti-PD- 1 antibody 10 days prior to ART initiation rapidly enhanced antiviral CD8 ${ }^{+} \mathrm{T}$ cell function and diminished IFN-stimulated genes. This resulted in faster viral suppression in plasma and better Th17 cell reconstitution in the rectal mucosa following ART initiation. PD-1 blockade during ART resulted in lower levels of cellassociated replication-competent virus. Following ART interruption, PD-1 antibody-treated animals showed markedly higher expansion of proliferating CXCR5'perforin ${ }^{+}$granzyme $\mathrm{B}^{+}$effector $\mathrm{CD8}^{+} \mathrm{T}$ cells and lower regulatory $T$ cells that resulted in better control of viremia. Our results show that PD-1 blockade can be administered safely with ART to augment antiviral CD8+ $T$ cell function and reduce the viral reservoir, leading to improved control of viral rebound after ART interruption.

Authorship note: CHM and LSC contributed equally to this work.

Conflict of interest: RA, CJF, and RRA are co-inventors of PD-1 technology that has been licensed to Genentech by Emory University.

Submitted: June 15, 2018 Accepted: August 17, 2018 Published: September 20, 2018

Reference information: JCI Insight. 2018;3(18):e122940. https://doi.org/10.1172/jici. insight.122940.

\section{Introduction}

Chronic viral infections such as lymphocytic choriomeningitis virus (LCMV), simian immunodeficiency (SIV), and human immunodeficiency virus (HIV) present with a characteristic footprint of immune exhaustion largely due to the presence of persistent antigen and unresolved immune activation. An important signature of immune dysfunction is increased and sustained expression of the coinhibitory receptor programmed cell death-1 (PD-1) on T cells, B cells, and activated monocytes (1-5). In chronic LCMV infection, antigenspecific $\mathrm{CD}^{+} \mathrm{T}$ cells maintain high levels of PD-1 expression that is associated with impaired polyfunctionality and proliferation. In vivo blockade of the interaction between PD-1 and its cognate ligands, either PD-L1 (B7-H1) or PD-L2 (B7-DC), markedly enhanced T cell responses and viral control (6). In chronically SIV-infected rhesus macaques (RMs), we and others demonstrated that in vivo blockade of PD-1 augmented the function of antiviral $\mathrm{CD}^{+} \mathrm{T}$ cells and $\mathrm{B}$ cells, leading to reduced plasma viremia and prolonged survival $(4,7)$. In $\mathrm{HIV}^{+}$individuals, PD-1 expression correlated with impaired $\mathrm{HIV}$-specific CD4 ${ }^{+}$and $\mathrm{CD}^{+} \mathrm{T}$ cell function, served as a predictor of disease progression, and in vitro blockade of PD-1/PD-L1 interactions enhanced $\mathrm{T}$ cell function $(1,5)$. Additionally, in vitro PD-1 blockade of cells from $\mathrm{HIV}^{+}$individuals restored antiviral CD4 ${ }^{+} \mathrm{T}$ helper cell capabilities that enhanced HIV-specific NK cell functionality (8). Thus, augmenting cellular immunity by targeting the PD-1 pathway is of significant interest to HIV-cure research, as antiviral $\mathrm{CD}^{+} \mathrm{T}$ cells are critical to control of HIV replication early after primary infection (9-14) and under suppressive antiretroviral therapy (ART) (15). In addition, there is considerable value in assessing the therapeutic effects of targeting the PD-1 axis during chronic HIV infection, as clinical outcomes in cancer patients have been significant with highly durable antitumor responses being observed (16-19). However, anti-PD-1 
therapy in $\mathrm{HIV}^{+}$individuals remain in the early stages and is currently being tested only in patients presenting with HIV and cancer comorbidities $(20,21)$, largely due to the lack of data on safety and therapeutic potential in a relevant animal model. It is also important to define the optimal dose and timing of PD-1 blockade with respect to ART in an effort to achieve maximal therapeutic benefit. Thus, there is a critical need for testing the safety and efficacy of anti-PD-1 therapy for HIV-infected individuals receiving ART using a pertinent preclinical animal model of SIV-infected ART-treated RMs.

Chronic HIV/SIV infection also presents with an aberrant increase in PD-1 expression on CD4 ${ }^{+} \mathrm{T}$ cells and this has been shown to highly correlate with plasma viremia and progressive disease $(1,22,23)$. Furthermore, several studies have demonstrated that $\mathrm{PD}-1^{+} \mathrm{CD} 4^{+} \mathrm{T}$ cells are highly infected, contribute to ongoing viral replication and production, and harbor a significant fraction of the HIV reservoir in patients under ART (24-27). This latent viral reservoir is the major obstacle to functional cure studies that aim to establish durable remission of HIV in the absence of ART, as the reservoir serves as the immediate source of viral recrudescence after treatment interruption. ART alone is not sufficient to eliminate the reservoir, as the half-life is estimated to be about 44 months, indicating that approximately 70 years of ART is required for complete viral eradication (28). Current strategies in the field aim to eliminate the viral reservoir by using latency-reversing agents (LRAs) to increase viral transcription in an effort to enhance immune surveillance and targeting of the reservoir, or to promote virus-induced cytopathic effects. These intended immunological outcomes are collectively referred to as the "shock and kill" approach $(29,30)$. Cure strategies also include the use of LRAs in combination with immune-based therapeutics such as vaccinations or checkpoint blockade to augment immune function and enhance targeting and clearance of infected cells. It is currently not known what impact the checkpoint inhibitor PD-1 blockade will have on HIV-1 transcription within latently infected $\mathrm{CD} 4^{+} \mathrm{T}$ cells during ART. It is possible that PD-1 blockade may result in increased transcription of NF- $\mathrm{KB}, \mathrm{NFAT}$, and other factors required for $\mathrm{T}$ cell activation and consequently reactivation of latent HIV (31-34). If true, PD-1 blockade will be uniquely positioned to simultaneously serve as an LRA and immune-enhancing agent to significantly improve antiviral immunity and substantially reduce the viral burden.

In the current study, we aimed to determine the safety and therapeutic potential of in vivo PD-1 blockade in combination with ART to enhance antiviral $\mathrm{CD}^{+} \mathrm{T}$ cell function and immune reconstitution and to destabilize the latent SIV reservoir in chronically SIV-infected RMs. Our findings demonstrate that PD-1 blockade can be administered safely in combination with ART to augment antiviral CD ${ }^{+} \mathrm{T}$ cell function, potentially disrupt the viral reservoir, and limit viral recrudescence after treatment interruption. These results have important implications for developing novel therapeutic interventions to administer in tandem with PD-1 blockade to better enhance antiviral immunity and viral clearance in an effort to achieve durable remission of HIV.

\section{Results}

Study design. RMs enrolled in this study were previously vaccinated with a DNA/MVA SIV vaccine and challenged intrarectally with SIVmac251 but failed to control infection (Supplemental Table 1; supplemental material available online with this article; https://doi.org/10.1172/jci.insight.122940DS1). The criteria for enrollment were based on plasma viral load cutoffs of between $1 \times 10^{4}$ and $5 \times 10^{5} \mathrm{RNA}$ copies $/ \mathrm{ml}$ of plasma at week 24 after infection (Supplemental Figure 1). Animals were assigned to either the therapeutic or control arm after standardizing for the set-point plasma viral loads at 24 weeks after infection, blood $\mathrm{CD}^{+} \mathrm{T}$ cell frequencies, rectal $\mathrm{CD} 4^{+} \mathrm{T}$ cell frequencies, and $\mathrm{GagCM} 9^{+} \mathrm{CD} 8^{+} \mathrm{T}$ cell frequencies for Mamu$\mathrm{A}^{*} 01 \mathrm{RMs}$ (Supplemental Figure 2A). Prior to the initiation of the trial, rectal central memory CD4 ${ }^{+} \mathrm{T}$ cell and SIV-specific $\mathrm{CD}^{+}$and $\mathrm{CD} 8^{+} \mathrm{T}$ cell frequencies were measured as baseline parameters and served to stratify treatment groups with comparable baseline immune parameters (Supplemental Figure 2B).

To determine the effects of PD-1 blockade in combination with ART and its potential to enhance antiviral immunity and reduce viral reservoirs, we performed PD-1 blockade in 2 phases: (I) prior to initiation of ART and (II) during suppressive ART. The aim for phase I of PD-1 blockade was to restore T cell functionality in the presence of viral antigen, which may consequently aid in the elimination of virus-infected cells. ART alone does not clear infected cells, but rather limits HIV/SIV replication by preventing production of new, infectious virions. However, by combining ART with PD-1 blockade, we aimed to simultaneously clear infected cells with functional antiviral $\mathrm{CD} 8^{+} \mathrm{T}$ cells as well as potentially diminish the reseeding of the established viral reservoir. To achieve this, we administered 5 doses of anti-PD-1 antibody (PD-1 $\mathrm{Ab}, 3 \mathrm{mg} / \mathrm{kg} /$ dose) intravenously on days $0,3,7,10$, and 14 to 10 SIVmac251-infected RMs between 24 
A
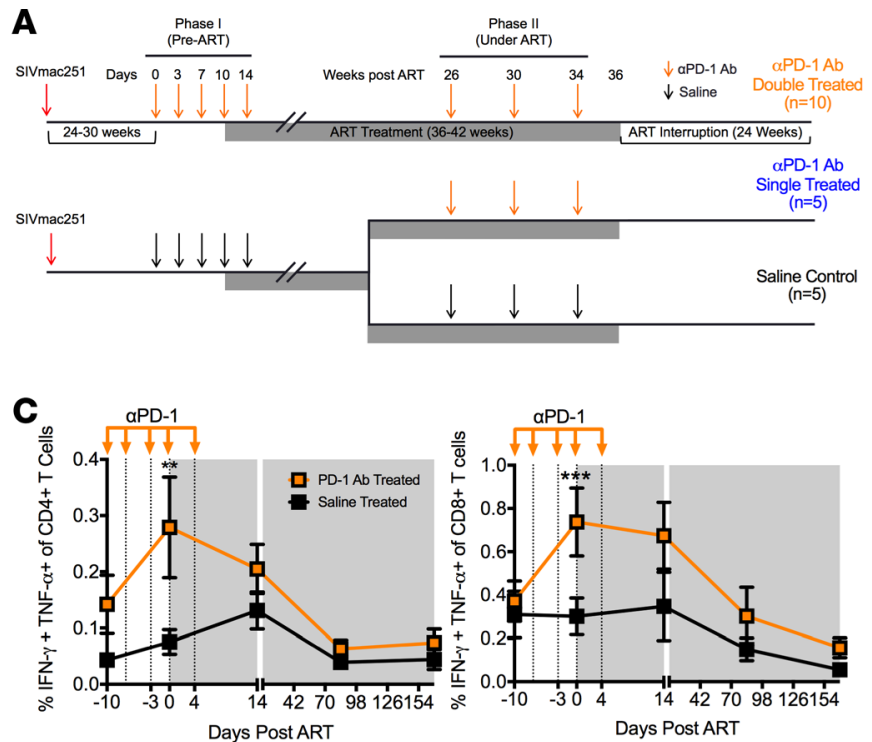

Days Post ART

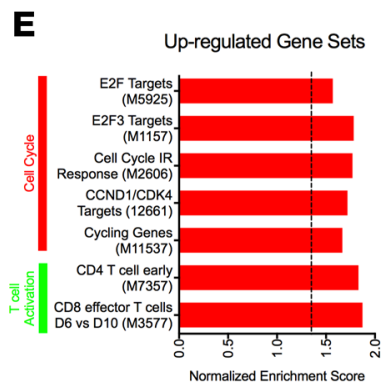

F
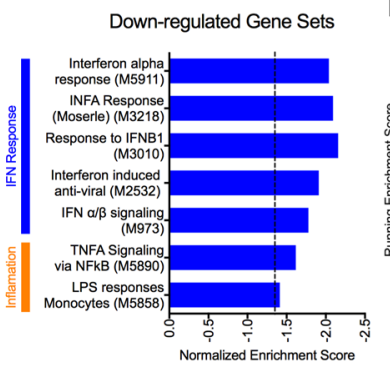

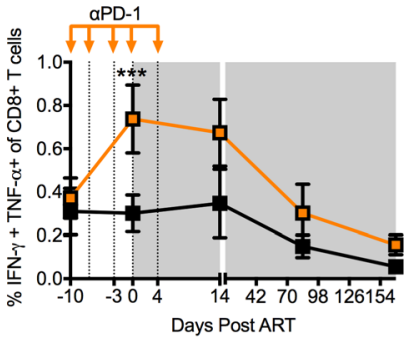

D

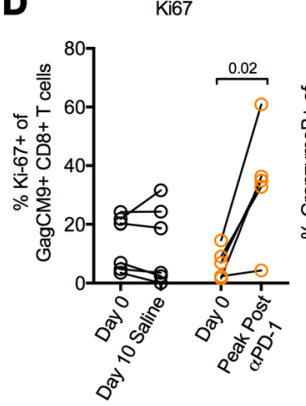

B

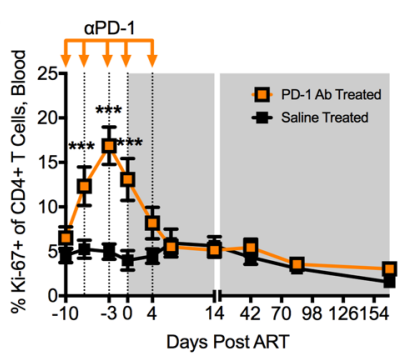

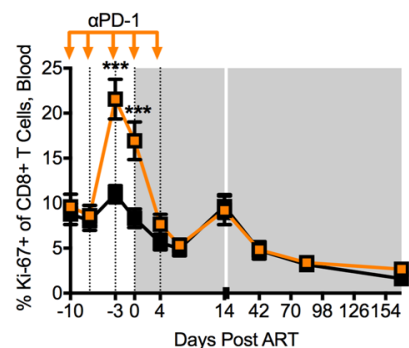

GranzymeB

CXCR5
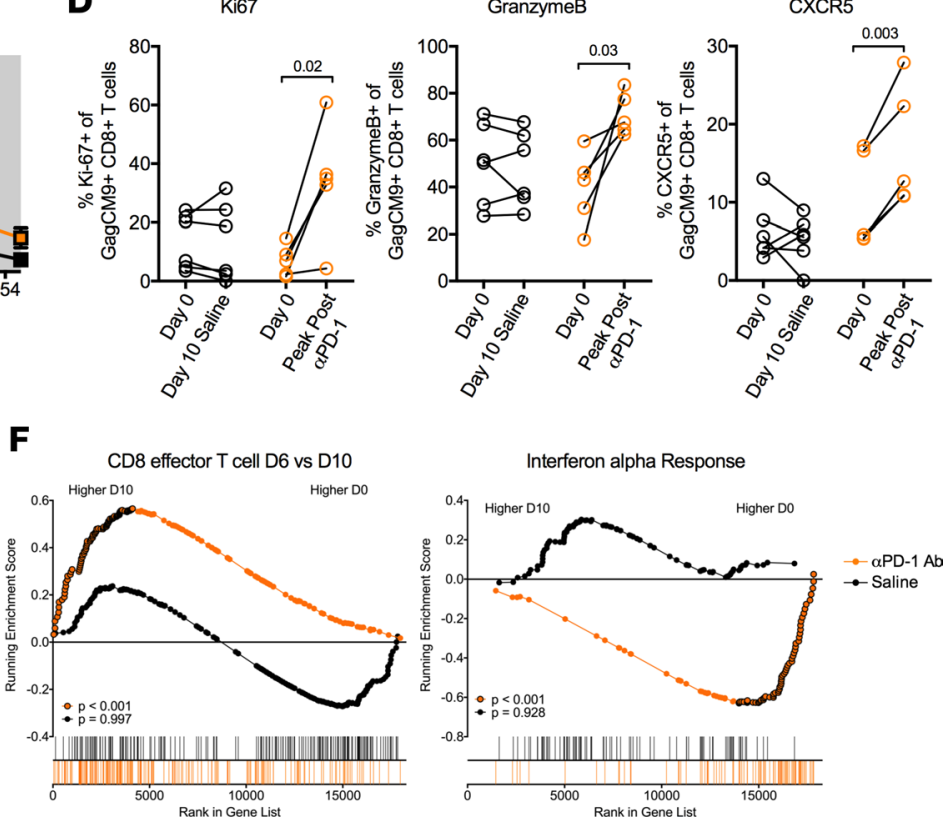

Figure 1. PD-1 blockade administered prior to ART (phase I) results in improved T cell functionality in SIV-infected RMs. (A) Schematic of PD-1 blockade strategy during phase I and II. (B) Frequency of Ki-67+CD4+ and Ki-67+CD8+ T cells in the blood. (C) Frequency of SIV Gag-specific and Env-

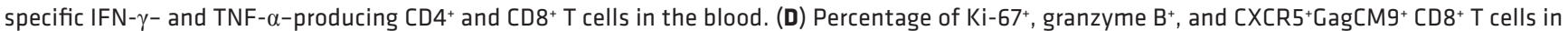
the blood (saline, $n=6$; PD-1 Ab treated, $n=5$ ). (E) Gene set enrichment analysis (GSEA) of RNA-Seq data from blood at day 10 compared with day 0 following PD-1 blockade during phase I (PD-1 Ab treated, $n=10)$. Normalized enrichment scores for select upregulated and downregulated gene sets depicted. Dashed line indicates normalized enrichment score cutoff of greater than 1.35 for upregulated gene sets and less than -1.35 for downregulated gene sets with a false discovery rate of less than 0.2. (F) GSEA plots comparing day 10 (D10) to day 0 (DO) of phase I for PD-1 Ab- and salinetreated $(n=5)$ groups. Leading-edge genes from gene sets are shown as black outlined dots. Shaded gray area depicts ART. Unfilled circles indicate values from Mamu-A*01 RMs. Data in $\mathbf{B}$ and $\mathbf{C}$ are shown as the mean $\pm \mathrm{SEM}$. ${ }^{* *} P<0.01$; ${ }^{* *} P<0.001$ by 2 -way ANOVA (B and $\mathbf{C}$ ) or 2 -tailed paired Student's $t$ test (D). $n=10$ per group unless otherwise noted.

and 30 weeks after infection (Figure 1A). As a control, we treated 10 SIVmac251-infected RMs with saline. To allow repeated administrations of PD-1 Ab for longer periods of time, we primatized our PD-1 Ab (clone EH12) by grafting humanized EH12 variable domains onto wild-type RM kappa and IgG4 constant regions. We confirmed the activity of the primatized antibody in vitro using $\mathrm{T}$ cells obtained from chronically SIV-infected RMs (Supplemental Figure 3A).

For phase II of the study, our goal was to determine if PD-1 blockade could cause reactivation of the latent viral reservoir and further expand virus-specific $\mathrm{CD} 8^{+} \mathrm{T}$ cells while animals were under $\mathrm{ART}$ in an effort to detect and clear infected cells. In the lymph nodes (LNs), a major site of the persistent viral reservoirs and where low-level replication of SIV may be occurring, exhausted CD8 $8^{+} \mathrm{T}$ cells may be unable to clear the infected cells and would benefit from the effects of PD-1 blockade. To determine these effects, the $10 \mathrm{RMs}$ given PD-1 Ab during phase I were again treated with PD-1 Ab (double treated) at 26-30 weeks following ART initiation. Three monthly infusions of PD-1 Ab were administered at $10 \mathrm{mg} / \mathrm{kg} / \mathrm{dose}$ (Figure 1A). To test the influence of PD-1 blockade administered only during suppressive ART, we split the 10 RMs from the saline group into 2 groups and gave 5 RMs PD-1 Ab (single-treated group) and saline to the remaining 5 RMs (saline control group) (Figure 1A). 
PD-1 blockade administered prior to ART improves T cell function. At day 3 following initiation of PD-1 blockade during phase I, plasma concentrations of the infused EH12 Ab reached $10-50 \mu \mathrm{g} / \mathrm{ml}$ that persisted until day 14 and declined by day 28, with one animal developing a measurable anti-EH12 response (Supplemental Figure 3, B and C). We initiated ART in all animals at day 10 after the initiation of PD-1 blockade. Following administration of PD-1 Ab, we observed a significant induction in the proliferation of circulating $\mathrm{CD}^{+}$and $\mathrm{CD} 8^{+} \mathrm{T}$ cells as measured by Ki-67 expression that peaked around day 7 (Figure 1B). Both central memory $\left(\mathrm{CD} 28^{+} \mathrm{CD} 95^{+}, \mathrm{Tcm}\right)$ and effector memory $\left(\mathrm{CD} 28^{-} \mathrm{CD} 95^{+}, \mathrm{Tem}\right) \mathrm{CD} 4^{+}$ and $\mathrm{CD}^{+} \mathrm{T}$ cells showed induction of Ki-67 (Supplemental Figure 3D). Additionally, we observed an increase in the frequency of $\mathrm{Ki}-67-$ expressing $\mathrm{CD}^{+}$and $\mathrm{CD}^{+} \mathrm{T}$ cells in the rectal mucosa of PD-1 Ab-treated RMs (Supplemental Figure 3E). Importantly, at day 10 of PD-1 blockade, we observed a significant increase in the frequency of SIV-specific IFN- $\gamma-$ and TNF- $\alpha$-producing CD4 ${ }^{+}$and CD8 ${ }^{+} \mathrm{T}$ cells (Figure 1C and Supplemental Figure 3F). A subset of animals in each group were Mamu-A*01+, which allowed us to assess the effects of PD-1 blockade on the function of SIV-specific CD8 ${ }^{+} \mathrm{T}$ cells utilizing the GagCM9 tetramer ( Tet $^{+}$cells). We found a significant increase in the proportion of $\mathrm{Tet}^{+}$ cells expressing Ki-67, granzyme B, and CXCR5, indicating that these cells are actively proliferating with improved cytolytic and lymphoid follicle homing potential (Figure 1D and Supplemental Figure 3G). We also found an increase in granzyme B expression on CXCR $5^{+} \mathrm{Tet}^{+}$cells $(P=0.02$, data not shown). The increase in CXCR5 expression is consistent with our recent report demonstrating that $\mathrm{CXCR} 5^{+} \mathrm{CD} 8^{+} \mathrm{T}$ cells serve as the predominant $\mathrm{CD} 8^{+} \mathrm{T}$ cell subset that responds to PD-1 blockade during chronic LCMV infection (35). As expected, following initiation of ART, the frequency of proliferating total and SIVspecific $\mathrm{T}$ cells decreased and this was associated with a decline in the frequency of SIV-specific CD $4^{+}$ and $\mathrm{CD}^{+} \mathrm{T}$ cells and viral antigen.

$P D-1$ blockade prior to ART results in global enrichment of effector $T$ cell responses and lowered immune activation. To determine the effects of PD-1 blockade on a global scale, we performed RNA sequencing (RNASeq) to compare gene signatures from the blood of $10 \mathrm{PD}-1 \mathrm{Ab}$-treated and 5 saline-treated animals at day 0 and day 10 of PD-1 blockade. Gene set enrichment analysis (GSEA) was performed to identify differentially expressed gene sets at day 10 compared to day 0 for each treatment group. We found significant enrichment in numerous gene sets related to immune cell phenotypes and cell signaling pathways that were not enriched in the saline-treated group (Figure 1E and Supplemental Tables 2-4). Gene signatures of note that were positively enriched in the PD-1 Ab treatment group were signatures associated with effector $\mathrm{CD}^{+} \mathrm{T}$ cell differentiation and $\mathrm{E} 2 \mathrm{~F}$ transcription factor targets related to cell cycling, indicating an impact of PD-1 blockade on effector $\mathrm{CD}^{+} \mathrm{T}$ cell function and proliferation (Figure 1, E and F, and Supplemental Figure 4, A and B).

PD-1 blockade during chronic infection also led to a downregulation of multiple gene sets associated with IFN-stimulated genes (ISGs) and TNF- $\alpha$ signaling (Figure 1, E and F, and Supplemental Figure 4, A and B). The downregulation of ISGs is notable since it has been shown to be associated with slower disease progression and lower viral reservoirs during chronic HIV/SIV infections (36-40). Moreover, these findings suggest that PD-1 blockade-induced dampening of ISGs may contribute to lower immune activation and better immune reconstitution after the initiation of ART. Reduction in overall immune activation is also indicated by negative enrichment of the TNF- $\alpha$ signaling via the NF- $\kappa \mathrm{B}$ gene set in the PD-1 Ab-treated RMs compared with saline-treated RMs (Figure 1E and Supplemental Figure 4A). Taken together, these gene sets indicate the unique potential of $\mathrm{PD}-1$ blockade to simultaneously enhance effector $\mathrm{CD} 8^{+} \mathrm{T}$ cell responses while dampening systemic immune activation observed during chronic pathogenic SIV infection (41).

We also sought to compare differences in gene expression of specific T cell-related genes (Supplemental Figure 4C) and observed an upregulation of transcripts associated with T follicular helper cells (Tfh) such as RGS10, CD200, FGF2, ICOS, TNFRSF4, and IL21, T helper 2 cells (Th2) such as IL4, and regulatory T cells (Tregs) such as CTLA4. Collectively, these results demonstrate that PD-1 blockade during chronic SIV infection resulted in a significant enhancement of genes associated with proliferation and effector $\mathrm{CD}^{+} \mathrm{T}$ cell response and dampening of a type I IFN response.

PD-1 blockade prior to ART improves viral suppression and immune reconstitution in the gut following ART initiation. Impressively, following initiation of ART, we observed an increase in the kinetics of viral suppression in the PD-1 Ab-treated RMs compared with saline-treated RMs. All animals in the PD- $1 \mathrm{Ab}$ group suppressed virus $(<100$ copies $/ \mathrm{ml}$ ) by 42 days, whereas saline-treated animals required up to 168 days to achieve the same level of suppression (Figure 2, A-C). Remarkably, $60 \%$ of RMs in the PD-1 blockade 
A

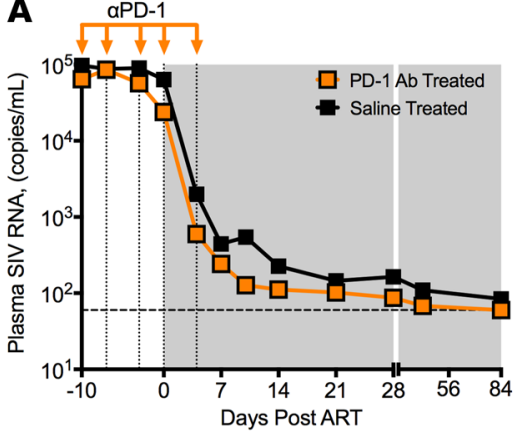

B

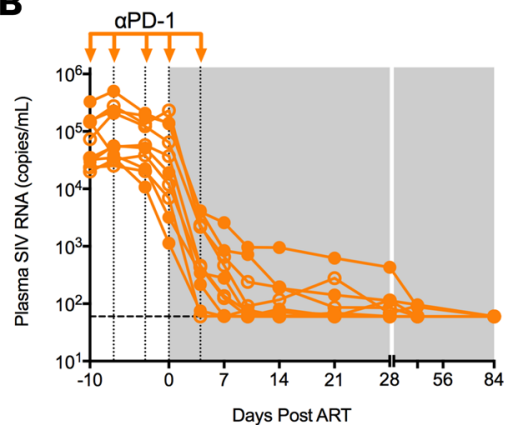

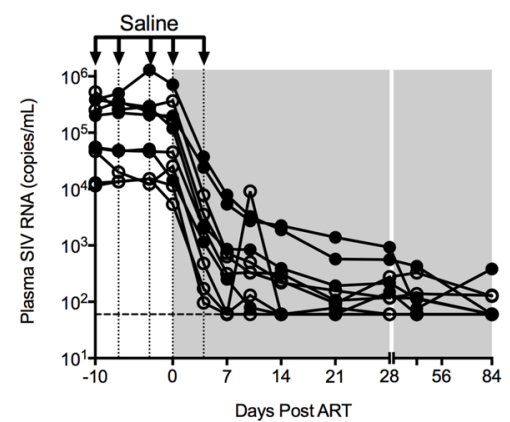
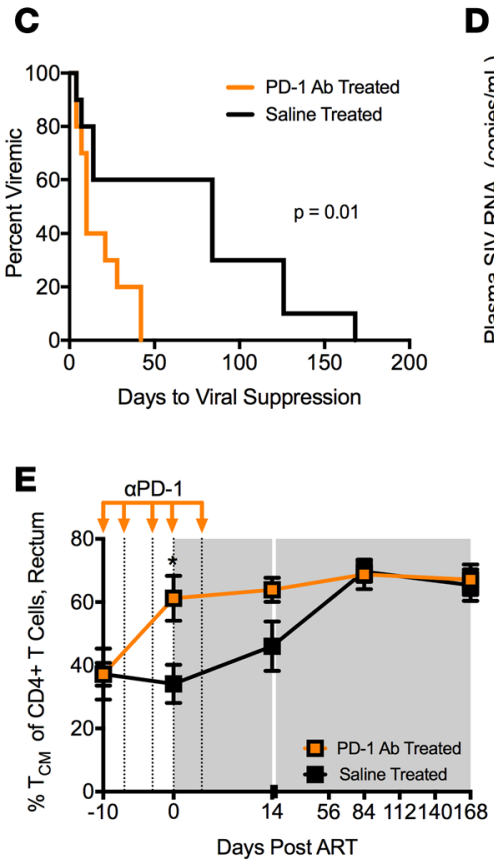

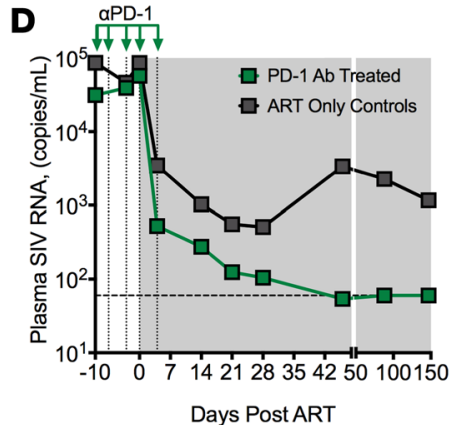

$\mathbf{F}$

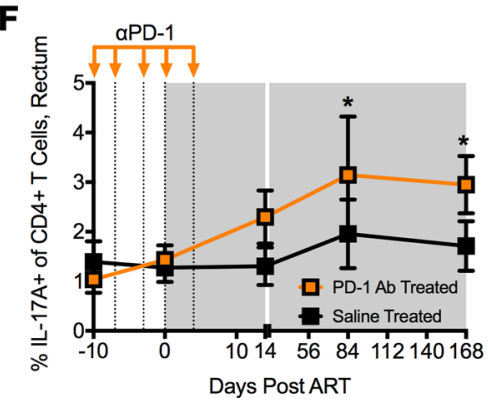

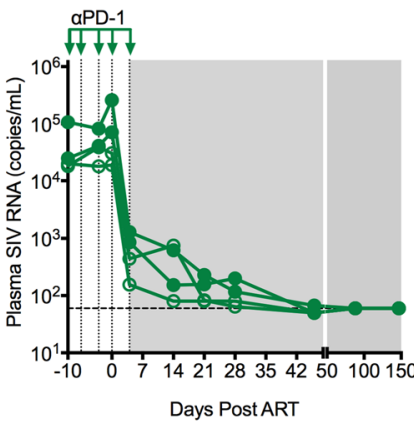

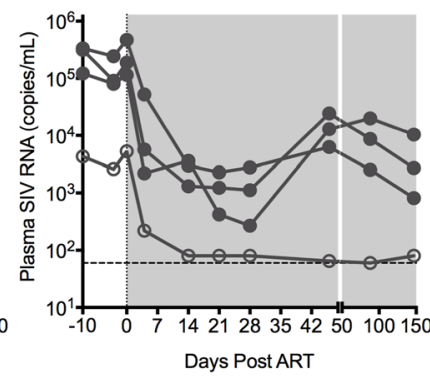

Figure 2. PD-1 blockade administered prior to ART results in improved viral suppression following ART initiation. Plasma SIV RNA viral loads (copies/ml) shown as (A) geometric mean for each group and (B) for individual RMs at initiation of anti-PD-1 Ab or saline infusion. Limit of detection is 60 copies/ml. (C) Kaplan-Meier curve of number of days until viral suppression (<100 copies/ml for at least 2 or more consecutive time points).

(D) Plasma SIV RNA viral loads (copies/ml) from chronically SIV-infected RMs administered humanized anti-PD-1 Ab or no Ab during ART initiation in a second study ( $n=4$ per group). (E) Frequency of Tcm $\left(C D 28^{+} C D 95^{+}\right)$CD4 $4^{+}$T cells in the rectum. (F) Frequency of IL-17A-producing CD4 $4^{+} T$ cells in the rectum after PMA and ionomycin stimulation (saline, $n=9$ ). (G) Frequency of neutrophils in lamina propria (LP) sections after 36 weeks of ART (saline, $n=5$; PD-1 Ab treated, $n=6$ ). Bars indicate the mean. Data were not collected for all animals. Shaded gray area depicts ART. Unfilled symbols indicate values from Mamu-A*01 RMs. Specific T cell frequency data are shown as mean \pm SEM. ${ }^{*} P<0.05$ by Mantel-Cox test (C), 2 -way ANOVA (E and $\mathbf{F}$ ), or 2-tailed Mann-Whitney test (G). $n=10$ per group unless otherwise noted.

group suppressed viremia by 10 days following ART initiation, whereas only $20 \%$ of animals in the saline group showed viral suppression at this time. Similar to these results, we observed enhanced viral suppression following PD-1 Ab administration and ART in another study, in which we treated SIVmac251-infected RMs with our humanized version of the PD-1 Ab (Figure 2D). The same time course for PD-1 blockade and ART regimen was utilized in this study; however, ART was initiated approximately 1 year after SIV infection. In this study, ART was only partially effective, as 3 of the 4 RMs in the ART-only group failed to control viremia (Figure 2D). Impressively however, all 4 animals in the PD-1 Ab group showed profound viral suppression, suggesting that PD-1 blockade synergized with ART even in long-term chronically SIV-infected RMs where ART suppression can be challenging. In both the pilot and present trial we utilized a suboptimal ART regimen when compared with current treatment modalities that include an integrase inhibitor $(15,42,43)$. It is, however, impressive that PD-1 blockade resulted in more rapid viral suppression when ART was initiated either at 26-30 weeks (current trial) or 1 year after infection (pilot trial). These data demonstrate that PD-1 blockade is effective in augmenting viral suppression when combined with ART. 
In addition to improved viral suppression, PD-1 blockade also resulted in early reconstitution of Tcm $\mathrm{CD}^{+} \mathrm{T}$ cells (Figure 2E) and better reconstitution of Th17 cells (Figure 2F) in the rectum, both of which have been shown to be critical for the long-term survival of SIV-infected RMs by helping to maintain the GI tract epithelial barrier function and decrease microbial translocation and hyperimmune activation (44-47). Consistent with this, at 36 weeks of ART, PD-1 blockade animals had lower frequencies of myeloperoxidase-expressing neutrophils in the lamina propria than the saline group, indicating that PD-1 blockade aided in the restoration of the GI tract epithelial barrier and reduced microbial translocation in the rectal mucosa in the treated animals (Figure 2G). Taken together, these data demonstrate that administration of PD-1 blockade for a short duration just prior to ART initiation improves the functional quality of SIVspecific T cell responses, enhances potency of ART, and better restores mucosal Th17 cells.

$P D-1$ blockade during ART results in $T$ cell proliferation and higher granzyme $B^{+} C X C R 5^{+} T$ cells in LNs. During phase II, we saw the concentrations of infused EH12 Ab in the serum peak at approximately $100 \mu \mathrm{g} / \mathrm{ml}$ at day 7 after each infusion, with 3 double-treated animals and 1 single-treated animal showing an anti-EH12 response (Supplemental Figure 5, A and B). Additionally, we performed complete blood count analysis as well as measured biochemical markers in the blood at day 0 , week 4 , and week 8 of phase II. We observed subtle yet significant changes in some biochemical parameters at week 4 and week 8 in the treated animals, although the values largely remained within the normal ranges expected for RMs (Supplemental Figures 6 and 7).

Following the first PD-1 Ab infusion under suppressive ART, we observed a significant increase in proliferation of $\mathrm{CD}^{+} \mathrm{T}$ cells in the double and single PD-1 Ab-treated groups (Figure $3 \mathrm{~A}$ ) and this proliferation was observed for both Tem and Tcm CD4 ${ }^{+} \mathrm{T}$ cells (Supplemental Figure 5C). CD8 ${ }^{+} \mathrm{T}$ cells and $\mathrm{Tet}^{+}$ cells also showed increased proliferation (Figure 3A). However, the proliferation was transient and sporadic following the second and third infusions (Supplemental Figure 5D). Intracellular cytokine analysis revealed an increase in SIV-specific CD8 ${ }^{+} \mathrm{T}$ cell responses in some animals after the first infusion (Supplemental Figure 5E). The $\mathrm{T}$ cell proliferation observed during phase II was much lower compared with proliferation observed during phase I. However, we think this is significant considering the low burden of SIV antigens during ART suppression. These results demonstrate that PD-1 blockade during suppressive ART can transiently induce proliferation of $\mathrm{CD} 4^{+} \mathrm{T}$ cells and total and antiviral $\mathrm{CD} 8^{+} \mathrm{T}$ cells.

To understand the influence of PD-1 blockade in the lymphoid tissue, we took LN biopsies 2 weeks after the final PD-1 Ab infusion and determined the granzyme $\mathrm{B}$ and CXCR5 expression on total $\mathrm{CD}^{+} \mathrm{T}$ cells and $\mathrm{Tet}^{+} \mathrm{CD} 8^{+} \mathrm{T}$ cells. We observed a higher magnitude of granzyme $\mathrm{B}^{+} \mathrm{CD} 8^{+} \mathrm{T}$ cells and $\mathrm{CXCR}^{+} \mathrm{Tet}^{+} \mathrm{CD} 8^{+} \mathrm{T}$ cells in PD-1 Ab-treated RMs compared with saline controls, suggesting these virusspecific and cytolytic cells may be localizing to sites known to harbor the viral reservoir (Figure $3 \mathrm{~B}$ ). We also observed an increased frequency of total CXCR $5^{+} \mathrm{CD} 4^{+} \mathrm{T}$ cells within the LNs, suggesting better preservation of follicular $\mathrm{CD}^{+} \mathrm{T}$ cells in the PD-1 Ab-treated animals (Figure 3B). These changes were not observed in the peripheral blood compartment, highlighting the tissue-specific effects of PD-1 blockade during ART (Supplemental Figure 5F).

PD-1 blockade during ART reduces the inducible viral reservoir and stimulates antiviral cellular response pathways in blood. To assess if PD-1 blockade impacted viral reservoirs, we isolated CD4 ${ }^{+} \mathrm{T}$ cells from the peripheral blood of RMs 2 weeks following the last PD-1 Ab infusion and cocultured them with CCR5 ${ }^{+}$CEM cells to detect viral outgrowth so as to indicate the presence of inducible replication-competent virus, similar to what has been previously described $(48,49)$. We found that PD-1 Ab-treated animals had measurably lower replication-competent virus assayed by viral RNA in the culture supernatant and p27 staining (Figure 3, C-E). Most PD-1 Ab-treated animals did not have measurable viral RNA within 500,000 purified CD4 ${ }^{+}$ $\mathrm{T}$ cells; therefore, we did not perform further dilutions.

To determine if PD-1 blockade was capable of reactivating latent viral reservoirs, we measured plasma viral RNA weekly after each infusion and observed transient increases (blips) in the double- and singletreated groups (Supplemental Figure 5, G and H). Though not significant due to limited power in the study and inability to sample daily, the frequency of transient increases in plasma viremia observed in the PD-1 $\mathrm{Ab}$-treated groups was higher compared with the saline alone, indicating a potential disruption of the viral reservoir. Taken together, these data demonstrate that PD-1 blockade administered under suppressive ART could potentially destabilize the inducible replication-competent viral reservoir and result in a lower viral burden within peripheral blood $\mathrm{CD} 4^{+} \mathrm{T}$ cells.

To further understand the global transcriptome changes that occur following PD-1 blockade during ART, we again compared gene signatures in the blood of PD-1 Ab-treated RMs from day 0 of first PD-1 
A CD4T cells

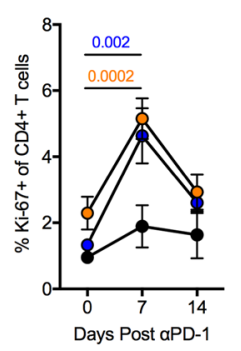

CD8 T cells

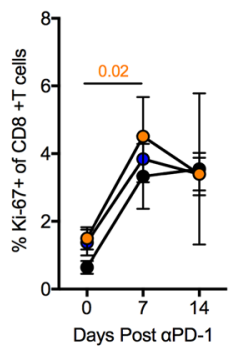

GagCM9+ CD8 T cells

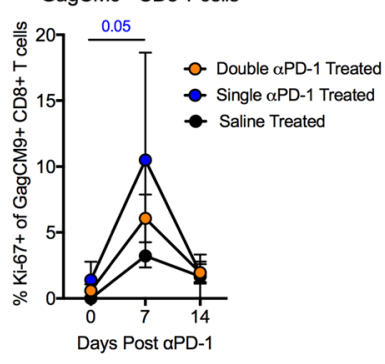

B

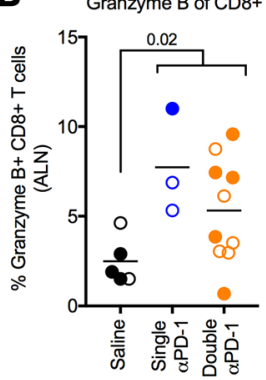

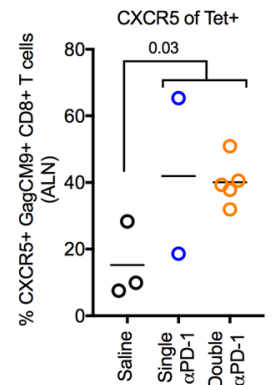

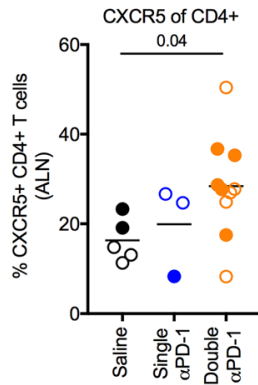

C

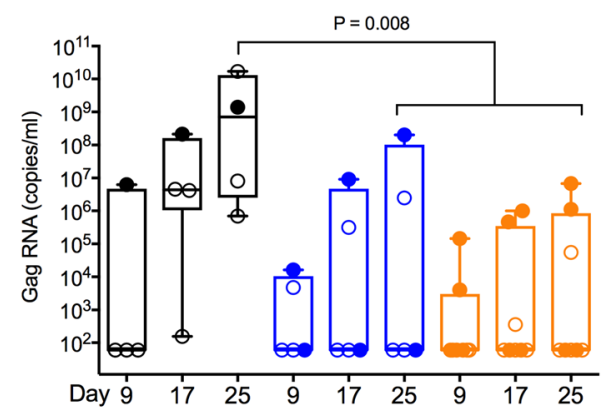

D

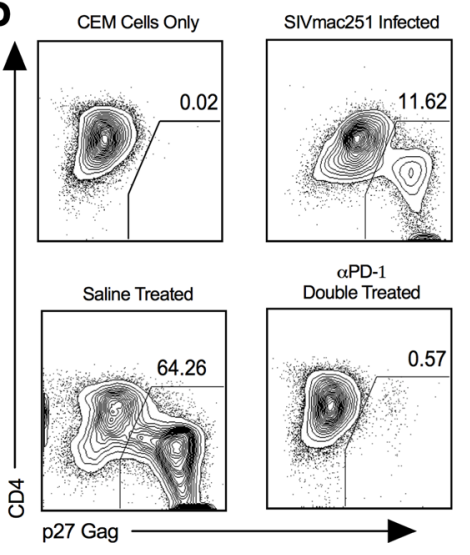

E

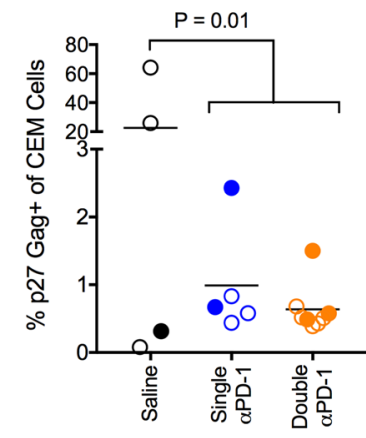

Figure 3. PD-1 blockade during suppressive ART (phase II) results in T cell proliferation and potential destabilization of the viral reservoir. (A) Ki-67 expression on $\mathrm{CD}^{+} \mathrm{T}$ cells, CD8 ${ }^{+} \mathrm{T}$ cells, and GagCM9+CD8 ${ }^{+} \mathrm{T}$ cells in blood after first PD-1 Ab infusion (right: saline, $n=2 ;$ single anti-PD-1 treated [ST], $n=3$; double anti-PD-1 treated [DT], $n=5$ ). Data are shown as the mean \pm SEM. (B) Frequency of granzyme $\mathrm{B}^{+} \mathrm{CD} 8^{+}$T cells (saline, $n=5 ; \mathrm{ST}, n=3 ; \mathrm{DT}, n=$ 10), CXCR5+CagCM9+CD8 ${ }^{+}$T cells (saline, $n=3 ; \mathrm{ST}, n=2 ; \mathrm{DT}, n=5$ ), and CXCR5 ${ }^{+}$CD4 ${ }^{+}$T cells (saline, $n=5 ; \mathrm{ST}, n=3 ;$ DT, $n=10$ ) in axillary lymph nodes (ALN) at 2 weeks after last PD-1 Ab infusion. Viral outgrowth assay on CD4+ $4^{+}$cells from blood of RMs 2 weeks after the last PD-1 Ab infusion. (C) SIV Gag RNA copies/ml assayed from culture supernatant at days 9, 17, and 25 of culture. (D) Representative p27 gag staining and (E) frequency of p27 gag ${ }^{+}$CEM cells from day 25 of viral outgrowth assay. For viral outgrowth assay, saline, $n=4 ; \mathrm{ST}, n=5 ; \mathrm{DT}, n=8$. Unfilled circles indicate values from Mamu-A*01 RMs. Bars indicate the mean. Exact $P$ values are shown. Two-way ANOVA (A), 2-tailed Mann-Whitney test (B and $\mathbf{C}$ ), 1-way ANOVA with Dunn's multiple-comparisons test (B, right), or 2-tailed Student's $t$ test (E) were used. One saline control animal was interrupted from ART early due to significant weight loss and therefore data are not available for this animal. Unless otherwise noted, saline, $n=4 ; \mathrm{ST}, n=5 ; \mathrm{DT}, n=10$.

$\mathrm{Ab}$ infusion under ART and 7 days after administration. Due to the limited sample availability for RNASeq during phase II, we combined the double- and single-treated animals into a single group to determine changes that occurred in response to PD-1 blockade treatment during ART (Figure 4A and Supplemental Tables 5-7). Gene sets for effector $\mathrm{CD}^{+} \mathrm{T}$ cells and cell cycling were again positively enriched after PD-1 blockade (Figure 4, A and B, and Supplemental Figure 8). Interestingly, ISGs were upregulated during this second phase of the PD-1 trial, which is in contrast to being downregulated during phase I (Figure 4, A and B, and Supplemental Figure 8). In addition, gene sets associated with IL-2/STAT5 signaling, which has been implicated in viral reactivation (50) as well as oxidative phosphorylation, also showed significant increases following PD-1 blockade (Figure 4A). Collectively, the transcriptome changes pointed to an active state of cell proliferation and transcription, which could promote reactivation of latent SIV in viral reservoirs resulting in potential increases in plasma viremia observed during phase II (Supplemental Figure 5, G and $\mathrm{H}$ ).

To determine if there was a core set of genes that responded to PD-1 blockade during both phase I and II, we curated a list of positively enriched gene sets that were significant during both phases and performed a leading-edge analysis (Supplemental Table 8). Our criterion for a signature gene was presence in at least 7 gene sets (Figure 4C). Many of the genes were related to cell cycling and proliferation, which is in line with the proliferative burst observed in T cells during both phases (Figure 1B and Figure $3 \mathrm{~A})$. These genes are considered the main drivers of the gene set enrichment observed for PD-1 blockade both in the absence and presence of ART.

$P D-1$ blockade enhances viral control in double-treated RMs after ART interruption. To better understand the therapeutic benefit of PD-1 blockade, we interrupted ART in all animals 2 weeks after the third 
A

Cell
Cycle

T cell
Activation
IFN
Response
Inflamation
Oxidative
Stress

\section{Up-regulated Gene Sets}

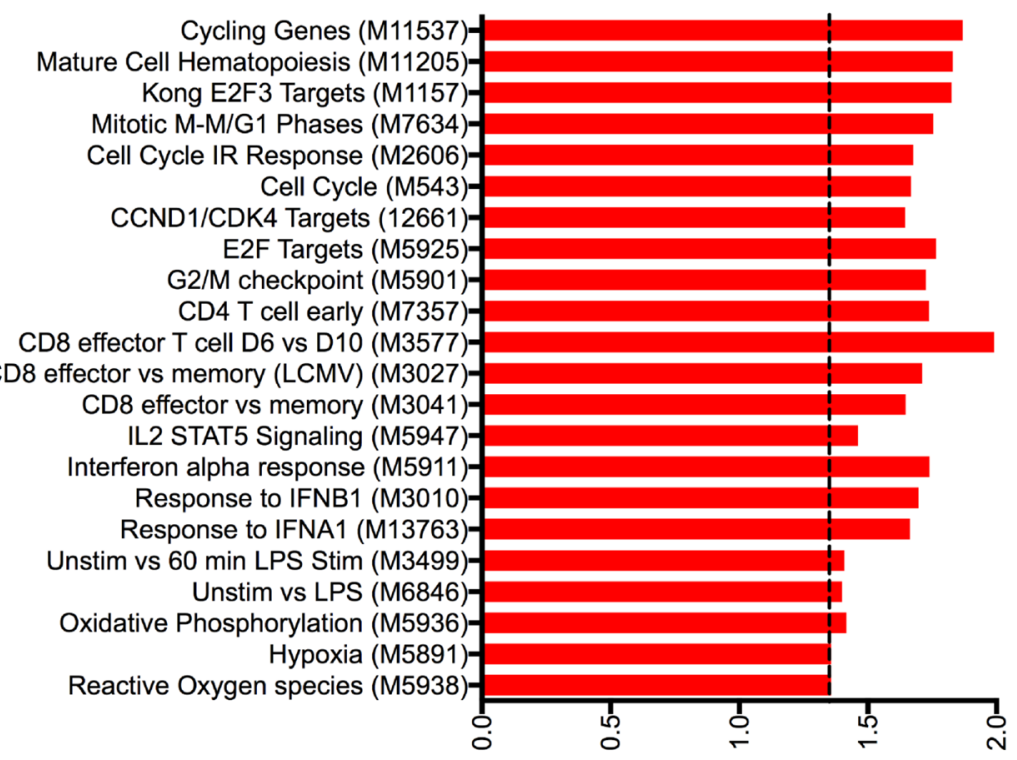

Normalized Enrichment Score
B CD8 effector T cell D6 vs D10

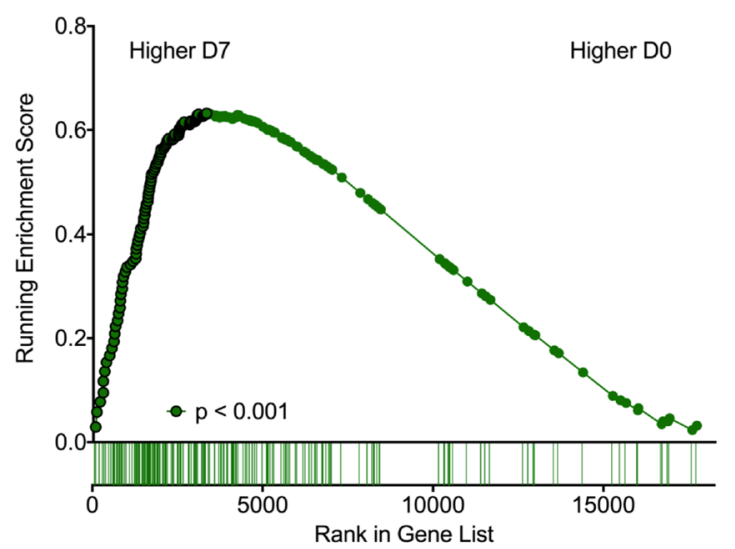

Interferon alpha Response

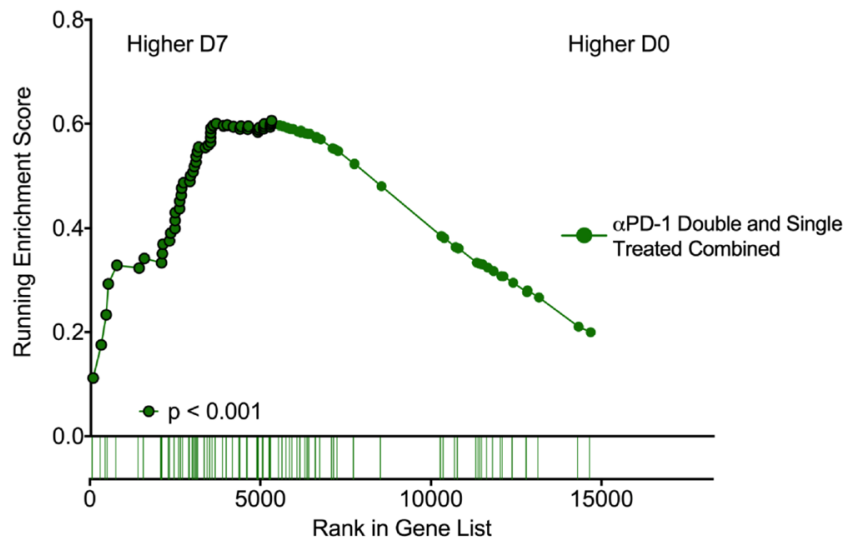

C

PD-1 Blockade Gene Signature

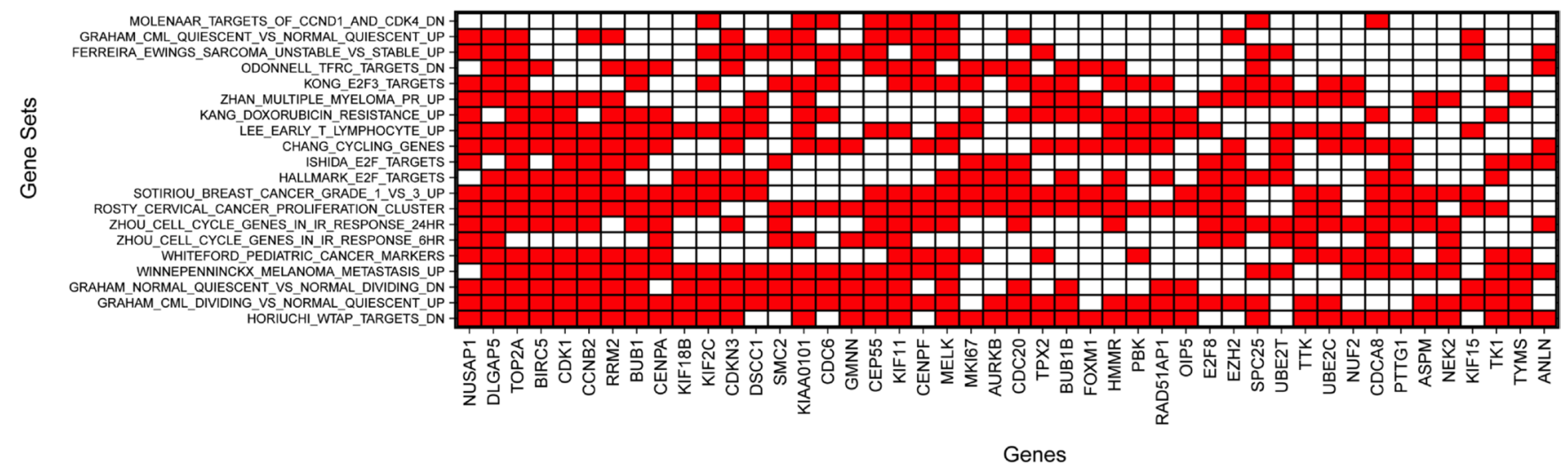

Figure 4. PD-1 blockade during suppressive ART (phase II) stimulates antiviral cellular response pathways. GSEA of RNA-Seq data from blood at day 7 compared with day 0 following first PD-1 Ab infusion for PD-1 Ab-treated animals during phase II. (A) Normalized enrichment scores for upregulated gene sets depicted. Dashed line indicates normalized enrichment score cutoff of greater than 1.35 with a false discovery rate of less than 0.2 . (B) GSEA plots comparing day 7 (D7) and day $\mathrm{O}(\mathrm{DO})$ of first PD-1 Ab infusion during phase II. Leading-edge genes from gene sets are shown as black outlined dots. Exact $P$ values are shown. $n=9$. (C) Leading-edge analysis was performed on positively enriched gene sets that were significant during both phase I and phase II. Genes present in at least 7 gene sets were considered core signature genes. Filled red boxes indicate presence of the gene in the respective gene set. 


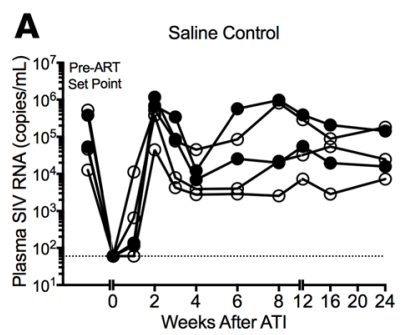

B

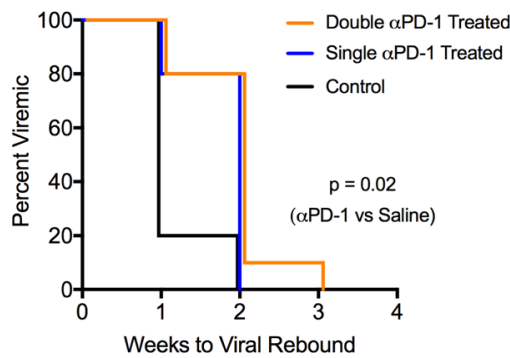

D

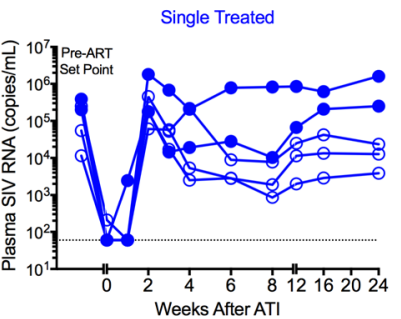

C Week 8
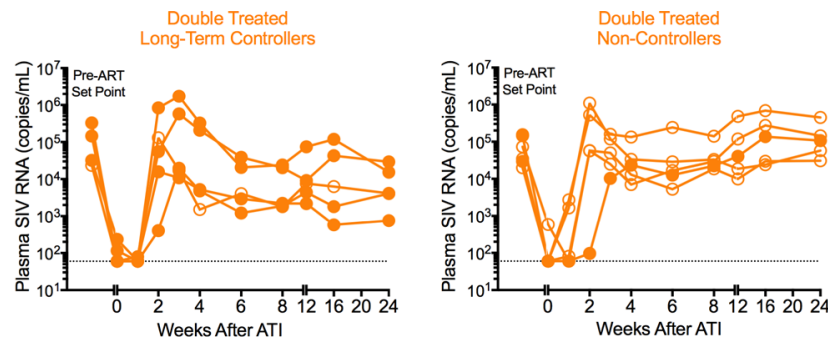

Week 12
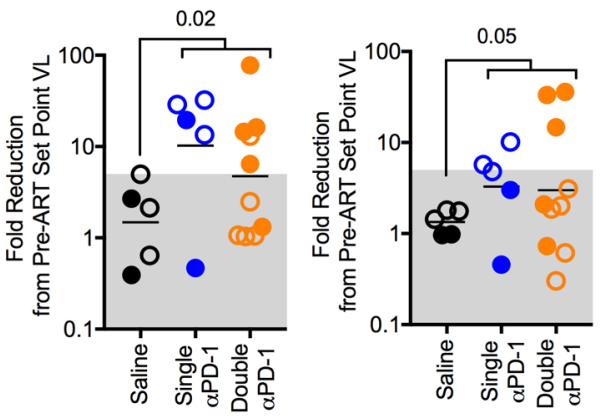

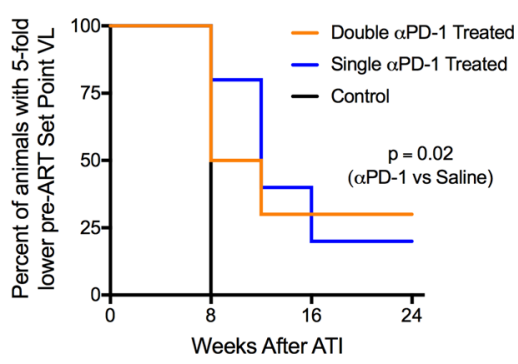

Figure 5. Enhanced viral control in PD-1 Ab-treated animals after ART interruption. (A) Plasma SIV RNA viral loads (copies/ml) in individual RMs at preART set point and after ART interruption (ATI) (day 0 of phase I and 36-42 weeks of ART treatment, respectively; see Figure 1A). Limit of detection is 60 copies/ml. (B) Kaplan-Meier curve showing number of weeks to viral rebound after ATI. Limit of detection is 60 copies/ml. (C) Fold reduction of viral loads (VL) from pre-ART set-point viral loads to weeks 8, 12, and 24 after ATI. Bars indicate geometric mean. (D) Kaplan-Meier curve showing number of weeks after ATI until viral loads reach within 5-fold levels of pre-ART viral loads. Unfilled circles indicate values from Mamu-A*01 RMs. Saline, $n=5$, single antiPD-1 treated, $n=5$; double anti-PD-1 treated, $n=10$. Mantel-Cox test (B and D) or 2-tailed Student's $t$ test with Welch's correction (C) were used.

PD-1 Ab infusion. We assessed the kinetics of viral rebound and various immune parameters until 24 weeks after treatment interruption. The virus rebounded in all animals by 3 weeks; however, consistent with lower viral reservoirs assessed by our viral outgrowth assay, we observed a significant 1-week delay in viral rebound in the $\mathrm{PD}-1 \mathrm{Ab}$-treated compared with the saline-treated group (Figure 5, A and $\mathrm{B}$ ). Virus rebounded in 4 of the 5 (80\%) saline-treated controls by 1 week, but only 3 of the $15(20 \%)$ PD-1 $\mathrm{Ab}$-treated animals showed viral rebound at this time. Additionally, we monitored the time until animals reached a set-point viral load similar to pre-ART levels (within 5-fold). PD-1 Ab-treated animals demonstrated a delay in approaching their pre-ART viral loads compared with saline control animals, with some PD-1 Ab-treated animals never attaining pre-ART levels (Figure 5, C and D). All saline controls reached their pre-ART viral loads by 8 weeks after ART interruption, showing no evidence of improved viral control. At 8 weeks following ART interruption, 5 of the 10 double-treated animals and 4 of the 5 single-treated animals showed 6- to 80-fold reduction of viral set points compared with preART levels (Figure 5C). At 24 weeks, 5 out of 10 double-treated animals sustained control, but only 1 of the 5 single-treated animals did. We believe this level of viral control in PD-1 Ab-treated animals is substantial since we initiated ART 6 months after infection, by which time it is expected the virus would have accumulated a significant number of escape mutations due to immune pressure.

PD-1 Ab-treated animals show enhanced polyfunctional CXCR $5^{+} C D 8^{+}$T cells and lower Tregs after ART interruption. Analysis of $\mathrm{CD}^{+}$and $\mathrm{CD} 4^{+} \mathrm{T}$ cell responses in the blood early after ART interruption showed a profound increase in the frequency of proliferating total $C D 8^{+} \mathrm{T}$ cells for $\mathrm{PD}-1 \mathrm{Ab}$-treated versus saline 
A

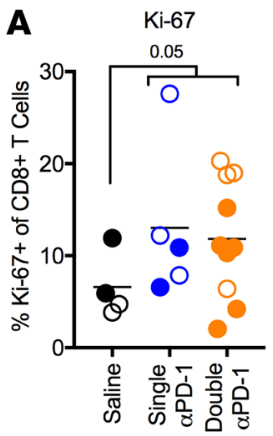

E

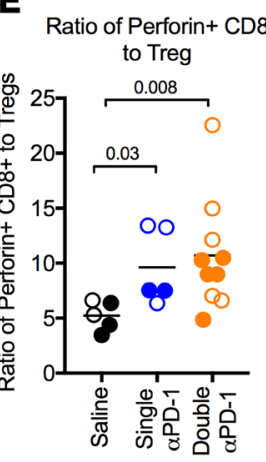

G

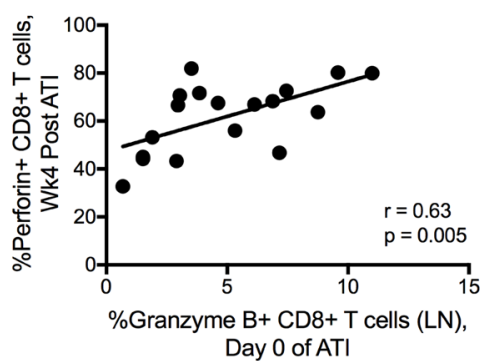

$\mathrm{Ki}-67+\mathrm{CXCR} 5+$

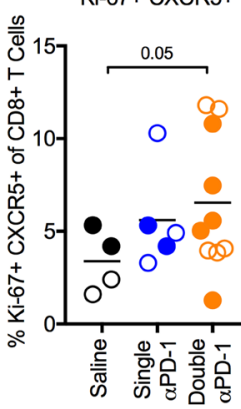

$\mathbf{F}$
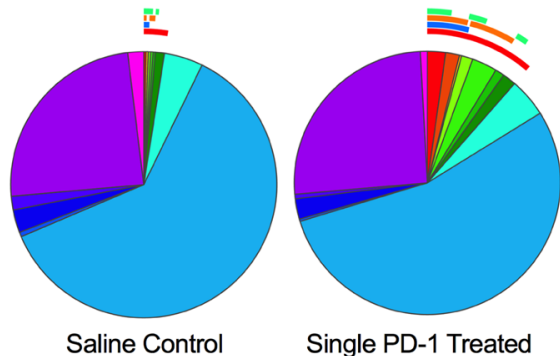

Single PD-1 Treated
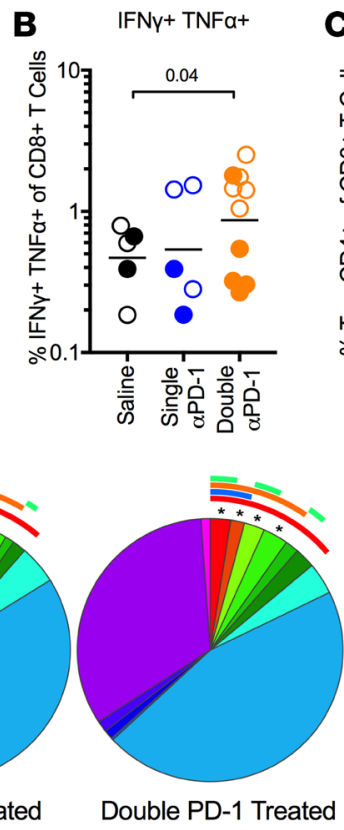
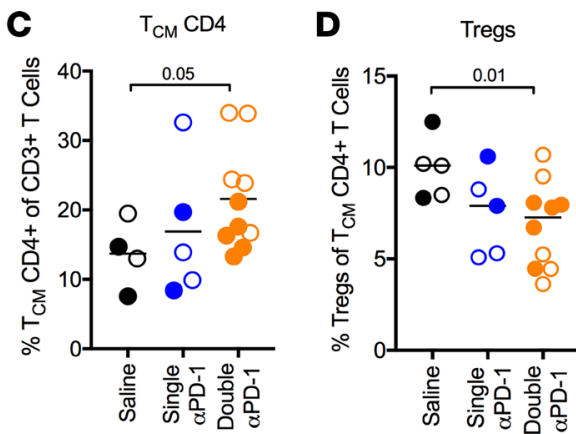

Pie Chart Arc Legend $\square$ CXCR5 +

$\square$ Granzyme B +

$\square$ Perforin +

$\square \mathrm{Ki}-67+$

Pie Chart Legend

CXCR5 $++++++++\ldots+\ldots$

Granzyme B + + + + - - - + + + + - -

Perforin ++--++-++--++

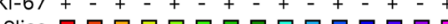

H

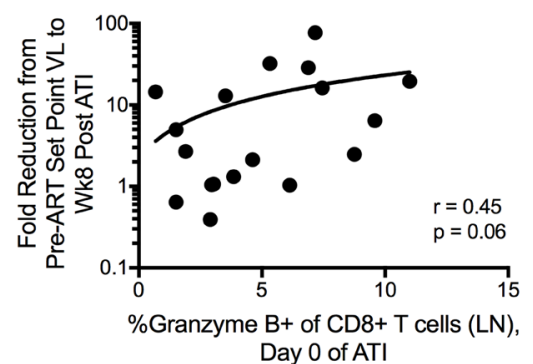

Figure 6. Enhanced immune responses in PD-1 Ab-treated animals after ART interruption (ATI). (A) Frequencies of Ki-67 ( 3 weeks after ATI; saline, $n$ $=4$ ), Ki-67+CXCR5 ${ }^{+}$(3 weeks after ATI; saline, $n=4$ ), and perforin ${ }^{+}$(4 weeks after ATI) CD8 ${ }^{+}$T cells in the blood. (B) Frequencies of SIV Gag-specific and Env-specific IFN- $\gamma$ - and TNF- $\alpha$-producing $C D 8^{+}$T cells in the blood at 4 weeks after ATI. Bars indicate the geometric mean. (C) Frequency of Tcm CD4 ${ }^{+} T$ cells as percentage of $\mathrm{CD}^{+} T$ cells in the blood at 2 weeks after ATI (saline, $\left.n=4\right)$. (D) Frequency of Tregs in the blood at 4 weeks after ATI. (E) Ratio of perforin ${ }^{+} C D 8^{+}$T cells to Tregs at week 4 after ATI. (F) Boolean analysis of marker expression of CXCR5, granzyme B, perforin, and Ki-67 on Tem CD8 ${ }^{+} T$ cells at 3 weeks after ATI. Correlations of frequency of granzyme B+CD8+ $T$ cells from lymph nodes (LNs) of animals at day 0 of ATI with (C) frequency of perforin ${ }^{+} \mathrm{CD} 8^{+} T$ cells at 4 weeks after ATI and (H) fold reduction of viral load (VL) from pre-ART set point to week 8 after ATI (single anti-PD- 1 treated, $n=$ 3). Unfilled circles indicate values from Mamu-A*01 RMs in A-E. Bars indicate the mean unless otherwise noted. ${ }^{*} P<0.05$ by 2 -tailed Student's $t$ test with Welch's correction (A-D), 2-tailed Mann-Whitney test (E and $\mathbf{F}$ ), or Spearman's rank-order correlations test $(\mathbf{G}$ and $\mathbf{H})$ were used. $r$ indicates the correlation coefficient. Unless otherwise noted, saline, $n=5$; double anti-PD-1 treated, $n=5$; single anti-PD-1 treated, $n=10$.

groups (Figure 6A and Supplemental Figure 9A). Proliferating CXCR $5^{+} \mathrm{CD} 8^{+} \mathrm{T}$ cells, perforin ${ }^{+} \mathrm{CD} 8^{+} \mathrm{T}$ cells (Figure 6A and Supplemental Figure 9A), and polyfunctional (IFN- $\gamma^{+} \mathrm{TNF}-\alpha^{+}$) SIV-specific CD8 ${ }^{+}$ $\mathrm{T}$ cells (Figure 6B and Supplemental Figure 9B) were found to be significantly increased in the double PD-1 Ab-treated compared with saline-treated RMs. The double PD-1 Ab-treated RMs also demonstrated a higher level of proliferating $\mathrm{CXCR} 5^{+} \mathrm{CD} 4^{+} \mathrm{T}$ cells (Supplemental Figure $9 \mathrm{C}$ ) and higher Tcm $\mathrm{CD}^{+} \mathrm{T}$ cells in the peripheral blood (Figure 6C and Supplemental Figure 9D). Remarkably, we also observed a decrease in the frequency of Tregs in the double-treated group compared with saline controls (Figure 6D). This resulted in 10 times higher levels of perforin ${ }^{+} \mathrm{CD}^{+} \mathrm{T}$ cells compared with Tregs in the double-treated group, compared with 5 times higher levels in saline controls (Figure 6E). Taken together, these data indicate enhanced immune function in the double-treated animals after ART interruption. A Boolean analysis performed on samples acquired at 3 weeks after treatment interruption revealed that the double-treated RMs generated higher frequencies of $\mathrm{CXCR} 5^{+} \mathrm{Tem} \mathrm{CD} 8^{+} \mathrm{T}$ cells, indicating 
enhanced cytolytic and lymphoid follicle homing potential of antiviral CD8 ${ }^{+} \mathrm{T}$ cells to eliminate virally infected cells within secondary lymphoid organs (Figure 6F and Supplemental Figure 9E). Interestingly, the majority of these immunological benefits were not significantly better in single-treated animals compared with controls, except for the increase in perforin ${ }^{+} \mathrm{CD}^{+} \mathrm{T}$ cells (Figure 6A and Supplemental Figure $9 \mathrm{~A}$ ) and the ratio of perforin ${ }^{+} \mathrm{CD}^{+} \mathrm{T}$ cells to Tregs (Figure $6 \mathrm{E}$ ). This could also be because of the smaller number of animals in this group.

Interestingly, the frequencies of $\mathrm{LN}$ granzyme $\mathrm{B}^{+} \mathrm{CD} 8^{+} \mathrm{T}$ cells on the day of ART interruption correlated with the frequencies of perforin ${ }^{+} \mathrm{CD} 8^{+} \mathrm{T}$ cells observed at 4 weeks after ART interruption (Figure 6G). LN granzyme B frequencies also trended toward a significant correlation with the viral load fold change at week 8 of ART interruption (Figure $6 \mathrm{H}$ ). The data indicate that the antiviral capacity of $\mathrm{CD}^{+} \mathrm{T}$ cells as detected in the LNs could play an important role in viral control after interruption of ART. These associations underscore the importance of understanding the cellular dynamics in the lymphoid tissues during blockade and how it will influence post-interruption responses. Taken together, these data demonstrate that PD-1 blockade combined with ART provides therapeutic benefit following ART interruption that includes a profound expansion of $\mathrm{CD}^{+} \mathrm{T}$ cells with increased cytolytic and lymphoid follicle homing potential.

\section{Discussion}

It is the goal of HIV-cure research to develop therapies that can both improve the antiviral immune response and simultaneously eliminate the viral reservoir. It is well understood that PD-1 regulates CD8 ${ }^{+} \mathrm{T}$ cell dysfunction during chronic HIV and SIV infection and our group has previously shown that blockade of the PD-1 pathway in ART-naive, chronically SIV-infected RMs is effective at improving the antiviral function of $\mathrm{CD}^{+} \mathrm{T}$ cells. Additionally, CD4 ${ }^{+} \mathrm{T}$ cells that express $\mathrm{PD}-1$ represent a significant fraction of the HIV and SIV reservoir. Therefore, in this current study, we aimed to test the safety and immune potential of $\mathrm{PD}-1$ blockade in conjunction with ART to improve $\mathrm{CD} 8^{+} \mathrm{T}$ cell function and reduce the viral reservoir that may lead to better control of SIV infection in the absence of ART. Our results show that PD-1 blockade can be safely and effectively combined with ART to enhance the function of antiviral immunity, improve Th17 cell reconstitution in the rectal mucosa, potentially subside mucosa-associated inflammation, and reduce the inducible peripheral blood viral reservoir. The results also demonstrate that the enhanced immune function mediated by PD-1 blockade can lead to higher and faster expansion of polyfunctional CXCR $5^{+} \mathrm{CD} 8^{+}$ $\mathrm{T}$ cells after ART interruption and contribute to better control of reemerging viremia. These results reveal important immunological and virological benefits as well as global transcriptome changes following PD-1 blockade and suppressive ART during chronic SIV infection that are important for the design of PD-1 blockade studies in HIV-infected ART-treated patients.

The mechanism by which ART suppresses viremia is by preventing the production of new virions and subsequent infection of new target cells. ART, however, does not eliminate infected cells. Therefore, the faster reduction in plasma viremia observed during phase I in the PD-1 Ab-treated animals could be a result of antiviral $\mathrm{CD} 8^{+} \mathrm{T}$ cells that emerged following PD-1 blockade with an enhanced ability to detect and clear productively infected cells. This is consistent with other studies demonstrating that $\mathrm{CD} 8^{+} \mathrm{T}$ cells play a pivotal role in controlling viremia even under conditions of suppressive ART therapy (15). PD-1 blockade could also have resulted in activation-induced cytopathic effects of infected CD4+ ${ }^{+}$cells, decreasing the overall half-life of an infected $\mathrm{CD} 4^{+} \mathrm{T}$ cell and resulting in a more rapid decrease in plasma viremia.

Impressively, PD-1 blockade prior to ART initiation resulted in a profound downregulation of ISGs within 10 days while improving $\mathrm{T}$ cell function. The downregulation of ISGs is unlikely due to a decrease in viremia since only a small fraction of animals showed a measurable decrease in viremia during this first 10 days following PD-1 blockade. HIV infection has been shown to induce ISGs in monocytes (51-53) and it is possible that PD-1 blockade on monocytes prevented ISG induction, leading to our observed downregulation of ISGs (Figure 1, E and F). This would suggest that cytolytic antiviral $\mathrm{T}$ cells that were increased during the same period are the main mediators of the rapid suppression of plasma viremia observed. The decrease in ISGs after PD-1 blockade remains notable, as lower levels of ISGs are associated with slower disease progression and reduced viral reservoirs during chronic HIV infection (36-39).

PD-1 blockade administered during suppressive ART had a less pronounced impact on $\mathrm{T}$ cell proliferation and function when compared with blockade given prior to the initiation of ART. In comparison with phase I, we observed a blunted increase in T cell proliferation for phase II; however, the baseline levels of $\mathrm{Ki}-67^{+} \mathrm{T}$ cells at day 0 of phase II were already lower than day 0 of phase I. Regardless of this, we still 
observed a 2- to 3-fold induction of proliferation after the first PD-1 Ab infusion during phase II. The very low levels of systemic viral antigen present during this phase of treatment, as well as a potential reduction in overall PD-1 expression due to lower level of persistent antigen and continuous ART (23), likely contributed to less significant levels of cell cycling and response to PD-1 blockade. It is possible that at sites enriched in SIV such as the GALT and secondary lymphoid tissues, immune responses could have emerged after blockade that were not captured in the periphery. This is supported in part by the increased frequencies of granzyme $\mathrm{B}^{+} \mathrm{CD} 8^{+}$and $\mathrm{CXCR} 5^{+} \mathrm{Tet}^{+} \mathrm{CD} 8^{+} \mathrm{T}$ cells in the $\mathrm{LNs}$, suggesting better homing potential of effector cells to the lymphoid tissues (site of viral reservoir) that was not observed systemically.

Our data suggest that PD-1 blockade administered during ART led to a potential destabilization of the viral reservoir. Transcriptional analysis during phase II indicated an upregulation of genes involved in effector $\mathrm{CD}^{+} \mathrm{T}$ cell activation, as expected, as well as cell cycling and type I IFN-response genes. It is possible that the increase in cell cycling genes was due to PD-1 blockade inducing reactivation of $\mathrm{PD}-1^{+} \mathrm{CD} 4^{+} \mathrm{T}$ cells harboring latent virus, thus leading to viral replication, and the observed transient increases in plasma viremia. This is consistent with a recent study demonstrating that PD-1 contributes to the establishment and maintenance of latently infected cells and blocking this pathway in HIV-infected individuals resulted in increased cell-associated HIV RNA in $\mathrm{CD}^{+}{ }^{+} \mathrm{T}$ cells (54). Subsequently, type I IFN genes were upregulated during phase II, suggesting that type I IFNs could be acting directly on latently infected CD4 ${ }^{+} \mathrm{T}$ cells to reactivate virus and result in the observed plasma blips or PD $-1^{+} \mathrm{CD} 4^{+} \mathrm{T}$ cells, in response to PD-1 blockade, have an altered intracellular environment skewing away from latency and towards active viral transcription. Although we observed blips in the saline-treated animals, these changes were small and occurred once in 2 of the 4 animals. Furthermore, due to the scheduling of sampling, destabilization of the reservoir and significant increases in plasma viremia may have occurred at earlier time points after infusion of PD-1 Ab and were therefore not captured by weekly sampling. Taken together, these data suggest that PD-1 blockade contributed to an overall improvement in the antiviral immune response and a potential destabilization of the latent reservoir. Due to the fact that proliferation of $\mathrm{T}$ cells was more profound in phase I compared with phase II and that both treatment groups exhibited viral outgrowth during phase II, it is possible that majority of immune-related effects of PD-1 blockade on the function of antiviral $\mathrm{CD} 8^{+} \mathrm{T}$ cells occurred during phase I and PD-1 blockade under suppressive ART largely effected PD- $1^{+} \mathrm{CD} 4^{+} \mathrm{T}$ cells.

Disruption of the PD-1 pathway can be achieved through blockade of the PD-1 receptor or its ligands PD-L1 or PD-L2. Anti-PD-L1 therapy is currently in clinical use for reversing immune exhaustion in patients with cancer and has led to responses and toxicity profiles similar to those observed with anti-PD-1 blockade (55). In chronic infection, PD-L1 is highly expressed on antigen-presenting cells, while PD-1 expression is increased and sustained on dysfunctional T cells. Anti-PD-1 blockade directly inhibits the interaction of PD-1 on T cells with its cognate ligand, while anti-PD-L1 blockade inhibits this interaction indirectly, without impacting PD-L2 engagement of PD-1. This would not allow for complete interruption of the PD-1 axis. Furthermore, the clone of Ab used for blockade could likely influence blockade efficacy. As the use of immune checkpoint inhibitors is investigated, it will be important to discern whether PD-1 or PD-L1 blockade results in differential outcomes and toxicities and to identify optimal clones for therapeutic use.

It is important to note that all RMs in the study had been chronically infected with SIVmac251 for 24-30 weeks, well into the chronic and progressive stage of infection before any intervention was administered. This length of chronic infection likely led to significant accumulations of viral mutations and viral escape in the animals. The chronicity and diversity of quasi-species in these animals would have made it difficult for any immune-based intervention to successfully eradicate the viral reservoir. That we were able to see improved antiviral $\mathrm{CD}^{+}$and $\mathrm{CD} 4^{+} \mathrm{T}$ cell responses, durable control of viremia under suboptimal ART, a delay in viral rebound, and differences in the set-point viral loads after ART interruption is encouraging, as individuals infected with HIV are now typically able to begin ART therapy during the acute or early chronic stages, before extensive chronic hyperimmune activation, exhaustion, and viral escape have occurred.

The range of immunological and therapeutic outcomes seen after PD-1 blockade treatment in our RMs is not surprising, as resistance to immune checkpoint inhibitor strategies in cancer patients has been well documented (56). Recently, Routy et al. described the influence of gut dysbiosis on PD-1 blockade efficacy (57). In particular, the authors show that use of antibiotics compromised PD-1 blockade efficacy in tumor models and cancer patients and the presence of certain commensal bacterial species improved responsiveness to blockade. In our previous study where PD-1 blockade was administered 
to chronically infected RMs in the absence of ART, we observed enhanced immune responses against gut-resident pathogenic bacteria (7). It is possible that PD-1 blockade enhanced responses against the microbiota in our current study and perturbed microbial composition in a way that could influence subsequent immune responses. Future analysis on the influence of the microbiota on PD-1 blockade efficacy in SIV infection would be informative to understand how the microbiota shapes immune dynamics during immune checkpoint blockade.

The results of this study provide preliminary evidence that PD-1 blockade can significantly augment antiviral immune responses, synergize with ART to increase the kinetics of viral suppression, and potentially destabilize latent viral reservoirs. Although the effects of PD-1 blockade suggest that this treatment may not stand alone as a monotherapy, the development and administration of therapeutic strategies in combination with PD-1 blockade could potentially lead to a significant decrease in the overall viral burden and potential remission of HIV. Combination immunotherapies that target additional checkpoint inhibitory receptors (CTLA-4, Tim-3, and Lag3) $(42,58)$ known to contribute to HIV persistence, in tandem with PD-1 blockade and ART would also have the potential to more significantly reverse dysfunction of exhausted cells and impact the stability of the viral reservoir. In vitro blockade of PD-1 and IL-10 signaling was shown to restore $\mathrm{CD}^{+} \mathrm{T}$ cell and NK cell functionality; thus, combination PD-1 and IL-10 blockade to improve immune cell reinvigoration during chronic infection would be of interest (8). Additionally, a recent study utilized a broad caspase inhibitor during acute infection and demonstrated better memory $\mathrm{CD}^{+} \mathrm{T}$ cell preservation and viral control (59). It would be interesting to investigate PD-1 therapy with the caspase inhibitor to assess whether synergy can be achieved. Additionally, coadministration of checkpoint inhibitors with TLR adjuvants, recently shown to impact post-ARTinterruption viral control (43), or costimulatory molecules could further enhance virus-specific immune responses aiding in viral clearance and immune control of HIV.

In conclusion, this study establishes the groundwork for preclinical studies to assess the efficacy of PD-1 blockade in ART-suppressed HIV-infected individuals. PD-1 blockade therapy is currently limited to end-stage cancer patients due to the risk versus benefits of coinhibitory blockade in HIV-infected patients tolerating long-term ART. Non-human primate studies such as these help establish the immunological and virological benefits of PD-1 blockade prior to human clinical trials. Our study demonstrates that PD-1 blockade and ART combination therapy can effectively reshape immune dynamics during chronic SIV infection, establishing a path forward in identifying optimal therapeutic strategies for HIV cure research.

\section{Methods}

Study group. Twenty-two Indian RMs (Macaca mulatta) chronically infected with SIVmac251 for 24-30 weeks with stable set-point viremia were used for the study. These macaques were previously vaccinated with a DNA/ MVA SIV vaccine and challenged intrarectally with SIVmac251 at a dose of $647 \mathrm{TCID}_{50}\left(1.25 \times 10^{7}\right.$ copies of viral RNA) but failed to control infection (Supplemental Table 1). Two animals of the single-treated group died due to thrombocytopenia and AIDS-related complications; thus, only 5 single PD-1 Ab animals were carried through to study completion. Data for the 2 macaques that died during the study are not presented. One saline control RM was interrupted from therapy prior to the initiation of phase II due to significant weight loss during suppressive ART and therefore data were not collected for that time period. RMs with plasma viral loads between $1 \times 10^{4}$ and $5 \times 10^{5} \mathrm{RNA}$ copies/ml in plasma at week 24 after infection were enrolled in the study. ART was initiated with the following combination of drugs: azidothymidine (AZT; $5 \mathrm{mg} / \mathrm{kg}$, twice daily), tenofovir (PMPA; $20 \mathrm{mg} / \mathrm{kg}$, daily), and emtricitabine (FTC; $30 \mathrm{mg} / \mathrm{kg}$, daily) administered subcutaneously; and Kaletra (12+3 mg/kg, twice daily) administered orally.

In vivo Ab treatment. Macaques were infused with $25 \mathrm{ml}$ of primatized anti-PD-1 Ab (clone EH12$2132 / 2133)$ in saline or $25 \mathrm{ml}$ of saline alone. The anti-PD-1 monoclonal $\mathrm{Ab}(\mathrm{mAb})$ is derived from the mouse anti-human EH12 mAb (60) and has a humanized variable heavy chain domain linked to a RM IgG4 and a humanized variable light chain domain linked to a RM kappa (RM constant regions were a gift from Rijan Wang and Keith Reimann, University of Massachusetts Medical School, Worcester, Massachusetts, USA). The EH12 mAb binds to RM PD-1 and blocks interaction between PD-1 and its ligands in vitro (2). Ab was produced in CHO cells using DHFR amplification (61), purified from culture supernatants by Protein G affinity chromatography, and verified to have an endotoxin level less than $2 \mathrm{EU} / \mathrm{mg}$. Anti-PD-1 Ab was administered intravenously at $3 \mathrm{mg} / \mathrm{kg}$ of body weight on days $0,3,7$, 10, and 14 during phase I and at $10 \mathrm{mg} / \mathrm{kg}$ monthly for 3 months during phase II. 
Isolation of mononuclear cells. Mononuclear cells were isolated from the blood, LNs, and rectal tissue of RMs, and flow cytometry analysis was performed as previously described $(2,4,62)$.

Abs. The following Abs were used: CXCR5 (MU5UBEE; eBioscience), CD3 (SP-34-2; BD Biosciences), GagCM9 tetramer (courtesy of the laboratory of Rafi Ahmed), CD28 (CD28.2; eBioscience), CD95 (DX2; BD Biosciences), CD279 (PD-1; EH12.2H7; Biolegend), CD8 (SK1; BD Bioscience), Ki-67 (B56; BD Biosciences), CD4 (L200; BD Biosciences), CD25 (BC96; Biolegend), granzyme B (GB11; BD Biosciences), IFN- $\gamma$ (B27; BD Biosciences), TNF- $\alpha$ (MAb11; BD Biosciences), perforin (Pf-80/164; BD Biosciences), IL17A (ebio64-Dec17; eBioscience), and FoxP3 (206D; Biolegend).

Phenotyping. Mononuclear cells were isolated from the blood and rectum as previously described (2, 4). Mononuclear cells were stained with LIVE/DEAD Near-IR Dead Cell stain (Life Technologies) and samples were acquired on an LSR-Fortessa (BD Biosciences). Cells stained for Treg cell phenotyping were stained for surface markers as described above and then fixed and permeabilized with an eBioscience FoxP3/Transcription Factor Staining Buffer Set according to the manufacturer's protocols.

Intracellular cytokine stimulation and staining. Mononuclear cells isolated from the blood and rectal samples were stimulated and stained as previously described $(2,4)$. Cells were stimulated with PMA (80 ng/ $\mathrm{ml})$ and ionomycin $(1 \mu \mathrm{g} / \mathrm{ml})$ for 4 hours and then surface stained with LIVE/DEAD Near-IR, anti-CD8, and anti-CD4 at room temperature for 20 minutes. Cells were acquired on an LSR-Fortessa and analyzed using FlowJo software (Tree Star).

Viral quantification. The SIV copy number in the plasma was determined by using quantitative real-time PCR as previously described (63). All samples were extracted and amplified in duplicate and the mean of the 2 values was then reported.

$R N A$-Seq. RNA-Seq analyses were conducted at the Yerkes NHP Genomics Core on PD-1 Ab-treated ( $n$ $=10)$ and saline-treated $(n=5) \mathrm{RMs}$ during phase I and total PD-1 Ab-treated RMs $(n=9)$ during phase II. RNA was collected and extracted from PAXgene tubes using on-column DNase digestion as described previously (38) and assessed for integrity and quantity using an Agilent Bioanalyzer (Agilent Technologies) and a NanoDrop 2000 spectrophotometer (Thermo Fisher Scientific). Hemoglobin transcript depletion was performed using Ambion human GLOBINclear kits (Ambion/Thermo Fisher Scientific) using $1 \mu \mathrm{g}$ of RNA as input, according to the manufacturer's instructions. Libraries were prepared using the Illumina TruSeq mRNA stranded kit as per the manufacturer's instructions. Briefly, 500-1,000 ng of Globin-depleted RNA was used for library preparation. ERCC synthetic spike-in controls 1 or 2 (Ambion) were added to each total RNA sample and processed in parallel. Amplified libraries were validated using the Agilent 4200 TapeStation and quantified using a Qubit fluorometer. Libraries were normalized, pooled, followed by clustering on a HiSeq 3000/4000 flowcell using the Illumina cBot. The clustered flowcell was then sequenced on the Illumina HiSeq 3000 system employing a single-end 101-cycle run, with multiplexing to achieve approximately 20 million reads per sample.

RNA-Seq statistical analyses. RNA-Seq data were aligned to the MacaM v7.8 assembly of the Indian RM genome (available at https://www.unmc.edu/rhesusgenechip/index.htm) alignment was performed with STAR v2.4.0g1 using the annotation as a splice junction reference. Transcripts were annotated using the MacaM v7.8.2 annotation. Transcript abundance was estimated using htseq-count v0.6.1p1 and differential expression analyses were performed using DESeq2. GSEA was performed using the desktop module available from the Broad Institute (https://www.broadinstitute.org/gsea/).

Viral outgrowth assays. Replication-competent SIV was cultivated from $\mathrm{CD}^{+} \mathrm{T}$ cells isolated from the peripheral blood of RMs at 14 days after the last PD- $1 \mathrm{Ab}$ infusion. Isolated CD4 ${ }^{+} \mathrm{T}$ cells $\left(5 \times 10^{5}\right)$ were preactivated with anti-CD3/CD28 beads (Miltenyi Biotec, non-human primate $\mathrm{T}$ cell expansion kit) for 24 hours and subsequently cocultured with CEM.NK ${ }^{\mathrm{R}}$ CCR $5^{+}$Luciferase $^{+} \mathrm{CD} 4^{+}$cells (CEM cells were a gift from David Evans, University of Wisconsin, Madison, Wisconsin, USA) in 24-well plates at a 1:1 ratio. Cells were cocultured for 25 days and supernatants were harvested at day 9, 17, and 25. SIV RNA in the supernatant was determined using qRT-PCR as previously described (63).

IHC and quantitative analysis of lamina propria from the rectal mucosa. IHC and quantitative analysis for the frequency of myeloperoxidase ${ }^{+}$neutrophils in the lamina propria was performed as previously described (64).

ELISA for anti-PD-1 (EH12) Ab serum titers. To measure the levels of infused PD-1 Ab, plates were coated with human PDCD1/PD-1 protein (Sino Biological, catalog number 10377-H-8H-50) in PBS, blocked, and incubated with different dilutions of plasma to capture the infused anti-PD-1 Ab. Bound Ab was detected using anti-RM IgG conjugated to horseradish peroxidase (HRP). Known amounts of anti-PD-1 $\mathrm{Ab}$ captured in the same manner were used to generate a standard curve. 
ELISA for anti-EH12 Ab response. To measure the level of Ab response generated against the infused EH12 Ab, plates were coated with the EH12 Ab $(2.5 \mu \mathrm{g} / \mathrm{ml})$ in PBS overnight. Dilutions (1:50) of plasma samples were incubated followed by mouse anti-RM IgG1-Biotin (7H11, NHP Reagent Resource) to detect bound $\mathrm{Ab}$. This detection $\mathrm{Ab}$ does not bind to the infused anti-PD-1 Ab due to its RM IgG4 heavy chain constant region. HRP-streptavidin was added, TMB substrate (KPL) was used for development, the reaction was stopped with $1 \mathrm{~N} \mathrm{H}_{3} \mathrm{PO}_{4}$, and absorbance read at $450 \mathrm{~nm}$.

Statistics. Statistical analyses were performed using Prism (version 7.0b; GraphPad). Statistical significance was determined using a 2-tailed paired Student's $t$ test for comparisons between matched time points for each animal. Two-way repeated-measures ANOVA with Dunnett's multiple-comparisons test was used to compare longitudinal data to baseline time points or Sidak's multiple-comparisons test was used to compare one time point to baseline values. One-way ANOVA with Dunn's multiple-comparisons test was used to compare 2 groups to a control group. Kaplan-Meier curves were compared using log-rank Mantel-Cox tests. Two-tailed unpaired Mann-Whitney test or Student's $t$ test with Welch's correction were performed for unmatched samples. Statistical analyses of global cytokine profiles were performed by partial permutation tests using SPICE software (NIAID, NIH) as previously described (65). $P$ values less than 0.05 were considered significant.

Study approval. RMs were housed at the Yerkes National Primate Research Center and were cared for under guidelines established by the Animal Welfare Act and the NIH Guide for the Care and Use of Laboratory Animals using protocols approved by the Emory University IACUC (Atlanta, GA).

Data availability. The RNA-Seq data set is available in the NCBI's Gene Expression Omnibus database (GEO GSE111435).

The data that support the findings of this study are available upon request from the corresponding author.

\section{Author contributions}

GHM and LSC performed experiments, analyzed data, and wrote the manuscript. GHM coordinated the study. GKT and SEB analyzed RNA-Seq data and discussed data interpretations. SH performed sample processing and data analysis. VV performed the pilot PD-1 blockade study using the humanized PD-1 Ab and contributed to the design of the current study. SSI performed initial SIVmac251 infections on RMs prior to treatment initiation. CD performed IHC analysis. JDE provided helpful discussions regarding IHC and data analysis. GJF synthesized primatized PD-1 Ab and provided helpful discussions regarding the study design. GHM, RA, and RRA contributed to the study design and data interpretation throughout the study. RRA supervised the study and wrote the manuscript.

\section{Acknowledgments}

We thank the veterinary staff at Yerkes for animal care; CFAR virology core for viral RNA and qPCR analysis; and CFAR immunology core for help with flow cytometry. We thank Rijan Wang and Keith Reimann at the University of Massachusetts Medical School for the RM constant regions of the IgG4 used in EH12 (antiPD-1) mAb. We thank David Evans at the University of Wisconsin for the CEM.NK ${ }^{\mathrm{R}}$ CCR $5^{+}$Luciferase $^{+}$CD ${ }^{+}$ cell line. We thank Nirav Patel at the Yerkes NHP Genomics Core for the sample processing, library preparation, and sequencing. This work was supported by the NIH grants R37 AI112787 and R01 AI074417 to RRA; YNPRC base grant, P51 RR00165; and Emory CFAR grant, P30 AI050409. This project has been funded in part with Federal funds from the National Cancer Institute, National Institutes of Health, under Contract No. HHSN261200800001E. The content of this publication does not necessarily reflect the views or policies of the Department of Health and Human Services, nor does mention of trade names, commercial products, or organizations imply endorsement by the U.S. Government.

Address correspondence to: Rama R. Amara, 954 Gatewood Road NE, Atlanta, Georgia 30329, USA. Phone: 001.4047278765; Email: ramara@emory.edu.

GHM's present address is: Ragon Institute of MGH, MIT, and Harvard, Cambridge, Massachusetts, USA. SI's present address is: Department of Pathology, Microbiology, and Immunology, California National Primate Research Center, University of California, Davis, Davis, California, USA. JDE's present address is: Vaccine and Gene Therapy Institute and Oregon National Primate Research Center, Oregon Health \& Science University, Beaverton, Oregon, USA. 
1. Day CL, et al. PD-1 expression on HIV-specific T cells is associated with T-cell exhaustion and disease progression. Nature. 2006;443(7109):350-354.

2. Velu V, et al. Elevated expression levels of inhibitory receptor programmed death 1 on simian immunodeficiency virus-specific CD8 T cells during chronic infection but not after vaccination. $J$ Virol. 2007;81(11):5819-5828.

3. Petrovas $\mathrm{C}$, et al. SIV-specific CD8 ${ }^{+} \mathrm{T}$ cells express high levels of PD1 and cytokines but have impaired proliferative capacity in acute and chronic SIVmac251 infection. Blood. 2007;110(3):928-936.

4. Velu V, et al. Enhancing SIV-specific immunity in vivo by PD-1 blockade. Nature. 2009;458(7235):206-210.

5. Trautmann L, et al. Upregulation of PD-1 expression on HIV-specific CD ${ }^{+} \mathrm{T}$ cells leads to reversible immune dysfunction. Nat Med. 2006;12(10):1198-1202.

6. Barber DL, et al. Restoring function in exhausted CD8 T cells during chronic viral infection. Nature. 2006;439(7077):682-687.

7. Dyavar Shetty R, et al. PD-1 blockade during chronic SIV infection reduces hyperimmune activation and microbial translocation in rhesus macaques. J Clin Invest. 2012;122(5):1712-1716.

8. Porichis F, et al. Immune checkpoint blockade restores HIV-specific CD4 T cell help for NK cells. J Immunol. 2018;201(3):971-981.

9. Jin X, et al. Dramatic rise in plasma viremia after CD8(+) T cell depletion in simian immunodeficiency virus-infected macaques. J Exp Med. 1999;189(6):991-998.

10. Schmitz JE, et al. Control of viremia in simian immunodeficiency virus infection by CD8 ${ }^{+}$lymphocytes. Science. 1999;283(5403):857-860.

11. Koup RA, et al. Temporal association of cellular immune responses with the initial control of viremia in primary human immunodeficiency virus type 1 syndrome. J Virol. 1994;68(7):4650-4655.

12. Ndhlovu ZM, et al. Magnitude and kinetics of $C D 8^{+} \mathrm{T}$ cell activation during hyperacute HIV infection impact viral set point. Immunity. 2015;43(3):591-604.

13. Borrow P, Lewicki H, Hahn BH, Shaw GM, Oldstone MB. Virus-specific CD8 ${ }^{+}$cytotoxic T-lymphocyte activity associated with control of viremia in primary human immunodeficiency virus type 1 infection. $J$ Virol. 1994;68(9):6103-6110.

14. Radebe $\mathrm{M}$, et al. Broad and persistent Gag-specific $\mathrm{CD} 8^{+} \mathrm{T}$-cell responses are associated with viral control but rarely drive viral escape during primary HIV-1 infection. AIDS. 2015;29(1):23-33.

15. Cartwright EK, et al. CD8(+) lymphocytes are required for maintaining viral suppression in SIV-infected macaques treated with short-term antiretroviral therapy. Immunity. 2016;45(3):656-668.

16. Robert C, et al. Nivolumab in previously untreated melanoma without BRAF mutation. N Engl J Med. 2015;372(4):320-330.

17. Weber JS, et al. Nivolumab versus chemotherapy in patients with advanced melanoma who progressed after anti-CTLA-4 treatment (CheckMate 037): a randomised, controlled, open-label, phase 3 trial. Lancet Oncol. 2015;16(4):375-384.

18. Ribas A, et al. Pembrolizumab versus investigator-choice chemotherapy for ipilimumab-refractory melanoma (KEYNOTE-002) a randomised, controlled, phase 2 trial. Lancet Oncol. 2015;16(8):908-918.

19. Eggermont AM, et al. Prolonged survival in stage III melanoma with ipilimumab adjuvant therapy. $N$ Engl J Med. 2016;375(19):1845-1855.

20. Wightman F, et al. Effect of ipilimumab on the HIV reservoir in an HIV-infected individual with metastatic melanoma. AIDS. 2015;29(4):504-506

21. Simeone E, et al. Immunological and biological changes during ipilimumab treatment and their potential correlation with clini$\mathrm{cal}$ response and survival in patients with advanced melanoma. Cancer Immunol Immunother. 2014;63(7):675-683.

22. Mylvaganam GH, et al. Diminished viral control during simian immunodeficiency virus infection is associated with aberrant PD-1hi CD4 T cell enrichment in the lymphoid follicles of the rectal mucosa. J Immunol. 2014;193(9):4527-4536.

23. Cockerham LR, et al. Programmed death- 1 expression on $\mathrm{CD}^{+}$and $\mathrm{CD} 8^{+} \mathrm{T}$ cells in treated and untreated HIV disease. AIDS. 2014;28(12):1749-1758.

24. Perreau M, et al. Follicular helper T cells serve as the major CD4 T cell compartment for HIV-1 infection, replication, and production. J Exp Med. 2013;210(1):143-156.

25. Banga R, et al. PD-1(+) and follicular helper T cells are responsible for persistent HIV-1 transcription in treated aviremic individuals. Nat Med. 2016;22(7):754-761.

26. Fukazawa Y, et al. B cell follicle sanctuary permits persistent productive simian immunodeficiency virus infection in elite controllers. Nat Med. 2015;21(2):132-139.

27. Chomont N, et al. HIV reservoir size and persistence are driven by $\mathrm{T}$ cell survival and homeostatic proliferation. Nat Med. 2009;15(8):893-900.

28. Siliciano JD, et al. Long-term follow-up studies confirm the stability of the latent reservoir for HIV-1 in resting CD4+ ${ }^{+}$cells Nat Med. 2003;9(6):727-728.

29. Kim Y, Anderson JL, Lewin SR. Getting the "kill" into "shock and kill": Strategies to eliminate latent HIV. Cell Host Microbe. 2018;23(1):14-26.

30. Margolis DM, Archin NM. Proviral latency, persistent human immunodeficiency virus infection, and the development of latency reversing agents. JInfect Dis. 2017;215(supp1_3):S111-S118.

31. Jiang G, et al. Synergistic reactivation of latent HIV expression by ingenol-3-angelate, PEP005, targeted NF-kB signaling in combination with JQ1 induced p-TEFb activation. PLoS Pathog. 2015;11(7):e1005066.

32. Chan JK, Greene WC. NF-кB/Rel: agonist and antagonist roles in HIV-1 latency. Curr Opin HIV AIDS. 2011;6(1):12-18.

33. DaFonseca S, et al. Purging the HIV-1 reservoir through the disruption of the PD-1 pathway. J Int AIDS Soc. 2010;13(Suppl 3):O15.

34. Pauken KE, et al. Epigenetic stability of exhausted T cells limits durability of reinvigoration by PD-1 blockade. Science. 2016;354(6316):1160-1165.

35. Im SJ, et al. Defining CD8 ${ }^{+} \mathrm{T}$ cells that provide the proliferative burst after PD-1 therapy. Nature. 2016;537(7620):417-421.

36. Sandler NG, et al. Type I interferon responses in rhesus macaques prevent SIV infection and slow disease progression. Nature. 2014;511(7511):601-605.

37. Jacquelin B, et al. Nonpathogenic SIV infection of African green monkeys induces a strong but rapidly controlled type I IFN response. J Clin Invest. 2009;119(12):3544-3555.

38. Bosinger SE, et al. Global genomic analysis reveals rapid control of a robust innate response in SIV-infected sooty mangabeys 
J Clin Invest. 2009;119(12):3556-3572.

39. Stacey AR, et al. Induction of a striking systemic cytokine cascade prior to peak viremia in acute human immunodeficiency virus type 1 infection, in contrast to more modest and delayed responses in acute hepatitis B and C virus infections. J Virol. 2009;83(8):3719-3733.

40. Cheng L, et al. Blocking type I interferon signaling enhances T cell recovery and reduces HIV-1 reservoirs. J Clin Invest. 2017;127(1):269-279.

41. Harris LD, et al. Downregulation of robust acute type I interferon responses distinguishes nonpathogenic simian immunodeficiency virus (SIV) infection of natural hosts from pathogenic SIV infection of rhesus macaques. J Virol. 2010;84(15):7886-7891

42. McGary CS, et al. CTLA-4+PD-1- Memory CD4 ${ }^{+} \mathrm{T}$ cells critically contribute to viral persistence in antiretroviral therapysuppressed, SIV-infected rhesus macaques. Immunity. 2017;47(4):776-788.e5.

43. Lim SY, et al. TLR7 agonists induce transient viremia and reduce the viral reservoir in SIV-infected rhesus macaques on antiretroviral therapy. Sci Transl Med. 2018;10(439):eaao4521.

44. Cecchinato V, et al. Altered balance between Th17 and Th1 cells at mucosal sites predicts AIDS progression in simian immunodeficiency virus-infected macaques. Mucosal Immunol. 2008;1(4):279-288.

45. d'Ettorre G, et al. Reconstitution of intestinal CD4 and Th17 T cells in antiretroviral therapy suppressed HIV-infected subjects: implication for residual immune activation from the results of a clinical trial. PLoS ONE. 2014;9(10):e109791.

46. Ortiz AM, et al. IL-21 and probiotic therapy improve Th17 frequencies, microbial translocation, and microbiome in ARV-treated, SIV-infected macaques. Mucosal Immunol. 2016;9(2):458-467.

47. Pallikkuth S, et al. Maintenance of intestinal Th17 cells and reduced microbial translocation in SIV-infected rhesus macaques treated with interleukin (IL)-21. PLoS Pathog. 2013;9(7):e1003471.

48. Micci L, et al. Interleukin-21 combined with ART reduces inflammation and viral reservoir in SIV-infected macaques. JClin Invest. 2015;125(12):4497-4513.

49. Shen A, et al. Resting $\mathrm{CD}^{+} \mathrm{T}$ lymphocytes but not thymocytes provide a latent viral reservoir in a simian immunodeficiency virus-Macaca nemestrina model of human immunodeficiency virus type 1-infected patients on highly active antiretroviral therapy. J Virol. 2003;77(8):4938-4949.

50. Jones RB, et al. A subset of latency-reversing agents expose HIV-infected resting CD4+ T-cells to recognition by cytotoxic T-lymphocytes. PLoS Pathog. 2016;12(4):e1005545.

51. Nasr N, et al. Mechanism of interferon-stimulated gene induction in HIV-1-infected macrophages. J Virol. 2017;91(20):e00744

52. Rempel H, Sun B, Calosing C, Pillai SK, Pulliam L. Interferon-alpha drives monocyte gene expression in chronic unsuppressed HIV-1 infection. AIDS. 2010;24(10):1415-1423.

53. Said EA, et al. Programmed death-1-induced interleukin-10 production by monocytes impairs $\mathrm{CD} 4^{+} \mathrm{T}$ cell activation during HIV infection. Nat Med. 2010;16(4):452-459.

54. Evans VA, et al. Programmed cell death-1 contributes to the establishment and maintenance of HIV-1 latency. AIDS. 2018;32(11):1491-1497.

55. Alsaab HO, et al. PD-1 and PD-L1 checkpoint signaling inhibition for cancer immunotherapy: Mechanism, combinations, and clinical outcome. Front Pharmacol. 2017;8:561.

56. Jenkins RW, Barbie DA, Flaherty KT. Mechanisms of resistance to immune checkpoint inhibitors. Br J Cancer. 2018;118(1):9-16.

57. Routy B, et al. Gut microbiome influences efficacy of PD-1-based immunotherapy against epithelial tumors. Science. 2018;359(6371):91-97.

58. Fromentin R, et al. CD4 ${ }^{+}$T cells expressing PD-1, TIGIT and LAG-3 contribute to HIV persistence during ART. PLoS Pathog. 2016;12(7):e1005761.

59. Laforge $\mathrm{M}$, et al. The anti-caspase inhibitor Q-VD-OPH prevents AIDS disease progression in SIV-infected rhesus macaques. J Clin Invest. 2018;128(4):1627-1640.

60. Dorfman DM, Brown JA, Shahsafaei A, Freeman GJ. Programmed death-1 (PD-1) is a marker of germinal center-associated T cells and angioimmunoblastic T-cell lymphoma. Am J Surg Pathol. 2006;30(7):802-810.

61. Naito M, et al. CD40L-Tri, a novel formulation of recombinant human CD40L that effectively activates B cells. Cancer Immunol Immunother. 2013;62(2):347-357.

62. Iyer SS, et al. Codelivery of envelope protein in alum with MVA vaccine induces CXCR3-Biased CXCR5 ${ }^{+}$and CXCR5- CD4 T cell responses in rhesus macaques. J Immunol. 2015;195(3):994-1005.

63. Amara RR, et al. Control of a mucosal challenge and prevention of AIDS by a multiprotein DNA/MVA vaccine. Science. 2001;292(5514):69-74.

64. Sereti I, et al. Decreases in colonic and systemic inflammation in chronic HIV infection after IL-7 administration. PLoS Pathog. 2014;10(1):e1003890.

65. Roederer M, Nozzi JL, Nason MC. SPICE: exploration and analysis of post-cytometric complex multivariate datasets. Cytometry A. 2011;79(2):167-174. 